\title{
DISCONTINUOUS GALERKIN DISCRETIZATION WITH EMBEDDED BOUNDARY CONDITIONS
}

\author{
P.W. HEMKER \\ $C W I$ \\ Kruislaan 413, 1098 SJ, Amsterdam, The Netherlands \\ E-mail: P.W.Hemker@cwi.nl \\ W. HOFFMANN \\ KdV Institute for Mathematics, University of Amsterdam \\ Plantage Muidergracht 24, 1018 TV Amsterdam, The Netherlands \\ E-mail: walter@science.uva.nl \\ M.H. VAN RAALTE \\ KdV Institute for Mathematics, University of Amsterdam \\ Plantage Muidergracht 24, 1018 TV Amsterdam, The Netherlands \\ E-mail: mvr@cwi.nl \\ Dedicated to Raytcho Lazarov on the occasion of his 60th birthday.
}

\begin{abstract}
The purpose of this paper is to introduce discretization methods of discontinuous Galerkin type for solving second-order elliptic PDEs on a structured, regular rectangular grid, while the problem is defined on a curved boundary. The methods aim at high-order accuracy and the difficulty arises since the regular grid cannot follow the curved boundary. Starting with the Lagrange multiplier formulation for the boundary conditions, we derive variational forms for the discretization of 2-D elliptic problems with embedded Dirichlet boundary conditions. Within the framework of structured, regular rectangular grids, we treat curved boundaries according to the principles that underlie the discontinuous Galerkin method. Thus, the high-order DGdiscretization is adapted in cells with embedded boundaries. We give examples of approximation with tensor products of cubic polynomials. As an illustration, we solve a convection-dominated boundary-value problem on a complex domain. Although, of course, it is impossible to accurately represent a boundary layer with a complex structure by means of cubic polynomials, the boundary condition treatment appears quite effective in handling such complex situations.
\end{abstract}

2000 Mathematics Subject Classification: 65N50; 65N99.

Keywords: discontinuous Galerkin discretization, structured grid, irregular boundary, embedded boundary.

\section{Introduction}

The purpose of this paper is to introduce methods of discontinuous Galerkin type for solving second-order elliptic PDEs on a structured, regular rectangular grid while the problem is 
defined on a curved boundary. The methods aim at high-order accuracy and the difficulty arises because the regular grid cannot follow the curved boundary.

Earlier, several techniques were proposed to handle boundary conditions on irregular, curvilinear boundaries. The most convenient is, of course, the FEM, where elements near the boundary are adapted to the shape of the boundary curve. Generally, this results in an unstructured grid. This relatively straightforward technique can be used with an arbitrarily high order of accuracy and produces good results.

In contrast, finite difference methods are usually applied on regular grids. Here, curved boundaries are treated by locally adapted finite differences as, e.g., the Shortley-Weller approximation [4, Sect.4.8]. Generally, such discretizations are not used for higher orders of accuracy.

A more recent technique for treatment of complex boundaries on orthogonal grids, in two or three dimensions, is the Embedded Curved Boundary (ECB) method. Here -usually in the context of the discretization of conservation laws- piecewise linear segments are embedded in the grid to represent the boundary. The method is also used, e.g., for solutions across interfaces $[7,8]$. In many cases the ECB method shows clear advantages compared to the traditional stair-step method [10] but no higher-order accuracy than order two can be expected.

A higher order may be obtained by Immersed Boundary Methods (IBM) [11-13,16], e.g., in pseudo-spectral codes [3], where the presence of the boundary within the computational domain is simulated by specifying a body force term, without altering the computational grid. This technique is very flexible as it allows for bodies and interfaces of almost arbitrary shape. The method is quite popular in situations with interfaces and rather complex geometries [9] and, e.g., elastic boundaries [15]. Usually the method is applied so as to maintain a secondorder accuracy (first order near the boundaries). However, fourth-order convergence rates are reported in [2], where the same methodology is used with PDEs for thin flexible membranes in an incompressible fluid domain.

In contrast with the above methods, we take the Lagrange multiplier formulation of the boundary conditions as a starting point, in the same manner as used in [14] or in the derivation of the discontinuous Galerkin discretization. Within the framework of structured, regular rectangular grids we introduce the treatment of curved boundaries in full agreement with the principles that lead to the discontinuous Galerkin method. We apply a high-order DG-discretization in the interior and adapt the method in cells with embedded boundaries. The order of approximation of the boundary condition corresponds with the accuracy of the DG-method. In the present paper we give examples of an approximation with tensor products of cubic polynomials.

In [6], we explain why the treatment of this cubic polynomial case is the basis for a higherorder approximation. In the DG discretization, the information exchange over the interior cell boundaries is restricted to the function values and normal fluxes. At the endpoints of an interval, the function values and fluxes are determined by four independent parameters, that correspond with the four degrees of freedom in the cubic polynomial approximation on a cell. A higher-order approximation can be achieved by additional bubble functions with vanishing values and derivatives at the cell boundary. In the multi-dimensional case, on a structured rectangular grid, the same principle holds with tensor-products for approximation.

For the treatment of embedded boundary conditions, we give in Section 2 of this paper an exposition of the weak forms used for different discretization alternatives. In Section 3 , we start with simple experiments in one and two dimensions to see the differences between 
the various methods. In Section 4, we identify the discrete function spaces in which the approximate solution is found. In the last section, we solve a convection-dominated equation on an irregular domain, partitioned into two cells only. We show how well, on this mesh, a complex problem can be solved with a piecewise cubic approximation.

\section{Weak forms for the Poisson equation}

\subsection{The Lagrange multiplier form for the embedded boundary problem}

To apply DG-methods for structured rectangular grids on complicated domains, we are interested in solving an elliptic second-order problem $L u=f$ on a fictitious open domain $\widehat{\Omega}$, which is larger than the open domain $\Omega$ on which the elliptic BVP is originally defined. The solution $u$ on $\Omega$ is determined by the Dirichlet boundary condition $u=u_{0}$ on $\partial \Omega$, the boundary of $\Omega$, and we want to discretize the problem on a fictitious domain $\widehat{\Omega} \supset \Omega$. For this purpose we assume that the solution $u$ on $\Omega$ allows a sufficiently smooth extension, $u$, defined on $\widehat{\Omega}$, solving $L u=f$. Of course, this excludes certain types of singularities near the boundary.

For the sake of simplicity, in this initial treatment we assume $\widehat{\Omega}$ to be a unit cube and we consider the Poisson equation with an embedded Dirichlet boundary condition as follows: let $\widehat{\Omega}$ be an open unit cube, with boundary $\partial \widehat{\Omega}$, which consists of two non-overlapping open sub-domains, $\Omega$ and $\widetilde{\Omega}$, such that

$$
\overline{\widehat{\Omega}}=\bar{\Omega} \cup \overline{\widetilde{\Omega}}, \quad \Omega \cap \widetilde{\Omega}=\emptyset,
$$

where $\widetilde{\Omega}$ is the fictitious part of the domain $\widehat{\Omega}$. We now consider the boundary value problem consisting of the Poisson equation defined on the whole of $\widehat{\Omega}$ and Dirichlet boundary conditions on $\partial \Omega$, the boundary of $\Omega$ :

$$
L u \equiv-\Delta u=f \text { on } \widehat{\Omega}, \quad u=u_{0} \text { on } \Gamma_{D}=\partial \Omega,
$$

under the assumption that the solution $u$ on $\Omega$ has a sufficiently smooth extension to $\widetilde{\Omega}$, satisfying the Poisson equation on the whole of $\widehat{\Omega}$.

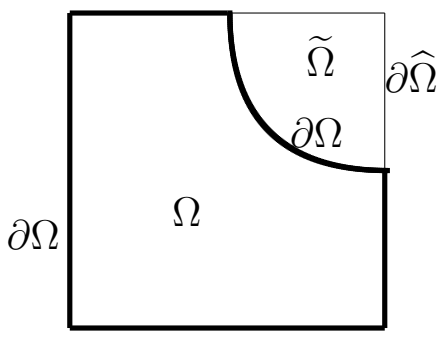

Figure 1. The domain of interest $\Omega$ and the fictitious part $\widetilde{\Omega}$ make the domain $\widehat{\Omega}=\Omega \cup \widetilde{\Omega}$.

To arrive at the corresponding weak formulation of the Poisson equation with an 'embedded' Dirichlet boundary condition, we multiply the left- and right-hand sides of (2.1) by a sufficiently smooth function $v$, and integrate over the domain $\widehat{\Omega}$, to get: find $u \in H^{1}(\widehat{\Omega})$ such that

$$
(\nabla u, \nabla v)_{\widehat{\Omega}}-\langle\mathbf{n} \cdot \nabla u, v\rangle_{\partial \widehat{\Omega}}=(f, v)_{\widehat{\Omega}} \quad \forall v \in H^{1}(\widehat{\Omega})
$$


under the constraint that $u=u_{0}$ on $\partial \Omega$. By the Lagrange multiplier theorem, the following formulation is equivalent to $(2.2)$ : find $u \in H^{1}(\widehat{\Omega})$ and $\bar{p} \in H^{-1 / 2}(\partial \Omega)$ such that

$$
\begin{aligned}
(\nabla u, \nabla v)_{\widehat{\Omega}}-\langle\mathbf{n} \cdot \nabla u, v\rangle_{\partial \widehat{\Omega}}+\langle\bar{p}, v\rangle_{\partial \Omega} & =(f, v)_{\widehat{\Omega}}, & & \forall v \in H^{1}(\widehat{\Omega}), \\
\langle q, u\rangle_{\partial \Omega} & =\left\langle q, u_{0}\right\rangle_{\partial \Omega}, & & \forall q \in H^{-1 / 2}(\partial \Omega) .
\end{aligned}
$$

We call this the Lagrange multiplier form of the embedded boundary problem. We see that if $u$ satisfies the Poisson equation (2.1) and the embedded Dirichlet boundary condition, the Lagrange multiplier $\bar{p}$ in (2.3) vanishes.

\subsection{The weak form for boundaries along gridlines}

In the classical case that $\widetilde{\Omega}=\emptyset$, we can combine the boundary terms in (2.3) to obtain

$$
\begin{aligned}
(\nabla u, \nabla v)_{\widehat{\Omega}}-\langle p, v\rangle_{\partial \widehat{\Omega}} & =(f, v)_{\widehat{\Omega}} & & \forall v \in H^{1}(\widehat{\Omega}), \\
\langle q, u\rangle_{\partial \Omega} & =\left\langle q, u_{0}\right\rangle_{\partial \Omega} & & \forall q \in H^{-1 / 2}(\partial \Omega),
\end{aligned}
$$

with $p=\mathbf{n} \cdot \nabla u-\bar{p}$ on $\partial \Omega=\partial \widehat{\Omega}$.

This leads to a hybrid form of (2.1) with Dirichlet BCs: find $u \in H^{1}(\widehat{\Omega})$ and $p \in$ $H^{-1 / 2}(\partial \Omega)$ such that

$$
(\nabla u, \nabla v)_{\widehat{\Omega}}-\langle p, v\rangle_{\partial \widehat{\Omega}}-\langle q, u\rangle_{\partial \Omega}=(f, v)_{\widehat{\Omega}}-\left\langle q, u_{0}\right\rangle_{\partial \Omega} \quad \forall v \in H^{1}(\widehat{\Omega}), q \in H^{-1 / 2}(\partial \Omega) .
$$

When $u$ satisfies $(2.1)$ we have $p=\mathbf{n} \cdot \nabla u$, the normal flux at the boundary $\partial \widehat{\Omega}$. Substituting this value for $p$ and replacing similarly the weighting function $q$ by $q=-\sigma \mathbf{n} \cdot \nabla v$, with $\sigma=1$ or $\sigma=-1$, we obtain the weak form used in DG-methods (viz., Baumann's and the symmetric DG-method respectively). Other DG-methods (viz., IPG, NIPG) are obtained by taking $q=-\sigma \mathbf{n} \cdot \nabla v-\mu v$ with parameters $\sigma$ and $\mu$. Thus, our DG weak form reads: find $u \in H^{1}(\widehat{\Omega})$ such that

$$
(\nabla u, \nabla v)_{\widehat{\Omega}}-\langle\mathbf{n} \cdot \nabla u, v\rangle_{\partial \widehat{\Omega}}+\sigma\langle\mathbf{n} \cdot \nabla v, u\rangle_{\partial \Omega}=(f, v)_{\widehat{\Omega}}+\sigma\left\langle\mathbf{n} \cdot \nabla v, u_{0}\right\rangle_{\partial \Omega}, \quad \forall v \in H^{1}(\widehat{\Omega}) .
$$

\subsection{The hybrid and the DG-form for the embedded boundary problem}

Not only the Lagrange multiplier form (2.3) can be used for the embedded boundary problem, we can also apply $(2.4)$ or $(2.5)$. In the case $\widetilde{\Omega} \neq \emptyset$, form $(2.4)$ reads: find $u \in H^{1}(\widehat{\Omega})$ and $p \in H^{-1 / 2}(\partial \widehat{\Omega})$ such that

$$
(\nabla u, \nabla v)_{\widehat{\Omega}}-\langle p, v\rangle_{\partial \widehat{\Omega}}-\langle q, u\rangle_{\partial \Omega}=(f, v)_{\widehat{\Omega}}-\left\langle q, u_{0}\right\rangle_{\partial \Omega} \quad \forall v \in H^{1}(\widehat{\Omega}), q \in H^{-1 / 2}(\partial \Omega),
$$

which we call the hybrid form of the interior boundary problem. In the case $\widetilde{\Omega} \neq \emptyset$, equation (2.5) is written: find $u \in H^{1}(\widehat{\Omega})$ such that

$$
(\nabla u, \nabla v)_{\widehat{\Omega}}-\langle\mathbf{n} \cdot \nabla u, v\rangle_{\partial \widehat{\Omega}}+\sigma\langle\mathbf{n} \cdot \nabla v, u\rangle_{\partial \Omega}=(f, v)_{\widehat{\Omega}}+\sigma\left\langle\mathbf{n} \cdot \nabla v, u_{0}\right\rangle_{\partial \Omega} \quad \forall v \in H^{1}(\widehat{\Omega}),
$$

which we call the $D G$-form (the Baumann-Oden weak form if $\sigma=1$ or the symmetric form if $\sigma=-1$ ) of the interior boundary problem. Note that this symmetric weak form is no longer symmetric if $\Omega \neq \widehat{\Omega}$. 


\section{Numerical experiments in one and two dimensions}

\subsection{Numerical experiments on one-dimensional problems}

To see the difference in practice, we first study the three weak forms $(2.3),(2.6)$ and $(2.7)$ for a simple one-dimensional problem. On the unit interval $\widehat{\Omega}=(0,1)$ we consider the Poisson equation with homogeneous Dirichlet boundary conditions:

$$
-\frac{d^{2} u}{d x^{2}}=f, \quad \text { on } \widehat{\Omega}, \quad \text { with } u(d)=0, \quad u(1)=0,
$$

where $d \in[0,1)$ and $\Omega=(d, 1)$. To discretize this problem, we take for test and trial spaces the $(p+1)$-dimensional space $S_{h}(\widehat{\Omega})=P^{p}(\widehat{\Omega}) \subset H^{1}(\widehat{\Omega})$, i.e., the space of polynomials of degree $\leqslant p$. We write for the approximate solution

$$
u_{h}=\sum_{0 \leqslant i \leqslant p} c_{i} \phi_{i}(x), \quad \phi_{i}(x) \in S_{h}(\widehat{\Omega}) .
$$

Further, we provide the boundary spaces $Q_{h}(\partial \widehat{\Omega}) \subset H^{-1 / 2}(\partial \widehat{\Omega})$ and $Q_{h}(\partial \Omega) \subset H^{-1 / 2}(\partial \Omega)$, with the trace of polynomials on the boundary, hence $Q_{h}(\partial \widehat{\Omega})=\left\{\psi_{0}(x)=\left.(1-x)\right|_{x=(0,1)}\right.$, $\left.\left.\psi_{1}(x)=\left.x\right|_{x=(0,1)}\right)\right\}$ and $\left.Q_{h}(\partial \Omega)=\left\{\psi_{0}(x)=\left.\frac{x-1}{d-1}\right|_{x=(d, 1)}, \psi_{1}(x)=\left.\frac{x-d}{1-d}\right|_{x=(d, 1)}\right)\right\}, d \in[0,1)$. Then we write for the approximation of the Lagrange multiplier

$$
p_{h}=\left.\sum_{0 \leqslant i \leqslant 1} a_{i} \psi_{i}(x)\right|_{x=0, d, 1}
$$

Because of the 1-D character of this example, the boundary values are parameterized by only two values for both $\partial \widehat{\Omega}$ and $\partial \Omega$. Given the approximating spaces, the three forms (2.3), (2.6) and (2.7) become:

(i) in the case of the Lagrange multiplier formulation: find $u_{h} \in S_{h}(\widehat{\Omega}), \bar{p}_{h} \in Q_{h}(\partial \widehat{\Omega})$ such that

$$
\begin{aligned}
\int_{0}^{1} u_{h}^{\prime} v_{h}^{\prime} d x & -\left[u_{h}^{\prime}(1) v_{h}(1)-u_{h}^{\prime}(0) v_{h}(0)\right]+\left[\bar{p}_{h}(1) v_{h}(1)-\bar{p}_{h}(d) v_{h}(d)\right] \\
& +\left[q_{h}(1) u_{h}(1)-q_{h}(d) u_{h}(d)\right]=\int_{0}^{1} v_{h} f d x \quad \forall v_{h} \in S_{h}(\widehat{\Omega}), q_{h} \in Q_{h}(\partial \widehat{\Omega})
\end{aligned}
$$

(ii) in the case of the hybrid form: find $u_{h} \in S_{h}(\widehat{\Omega}), p_{h} \in Q_{h}(\partial \Omega)$ such that

$$
\begin{gathered}
\int_{0}^{1} u_{h}^{\prime} v_{h}^{\prime} d x-\left[p_{h}(1) v_{h}(1)-p_{h}(0) v_{h}(0)\right]-\left[q_{h}(1) u_{h}(1)-q_{h}(d) u_{h}(d)\right] \\
=\int_{0}^{1} v_{h} f d x, \quad \forall v_{h} \in S_{h}(\widehat{\Omega}), \quad q_{h} \in Q_{h}(\partial \widehat{\Omega}) ;
\end{gathered}
$$


(iii) whereas the $D G$-formulation reduces to: find $u_{h} \in S_{h}(\widehat{\Omega})$ such that

$$
\begin{aligned}
\int_{0}^{1} u_{h}^{\prime} v_{h}^{\prime} d x- & {\left[u_{h}^{\prime}(1) v_{h}(1)-u_{h}^{\prime}(0) v_{h}(0)\right]+\sigma\left[v_{h}^{\prime}(1) u_{h}(1)-v_{h}^{\prime}(d) u_{h}(d)\right] } \\
& =\int_{0}^{1} v_{h} f d x \quad \forall v_{h} \in S_{h}(\widehat{\Omega}) .
\end{aligned}
$$

As a first experiment, we check if the three discrete forms (3.2), (3.3) and (3.4) will lead to the exact solution, when we choose $f(x)=x$ in (3.1) and $d=1 / 2$, and if we take $S_{h}(\widehat{\Omega})=P^{3}(\widehat{\Omega})$. The result is shown in Fig. 2. It appears that all three formulations compute the exact solution.

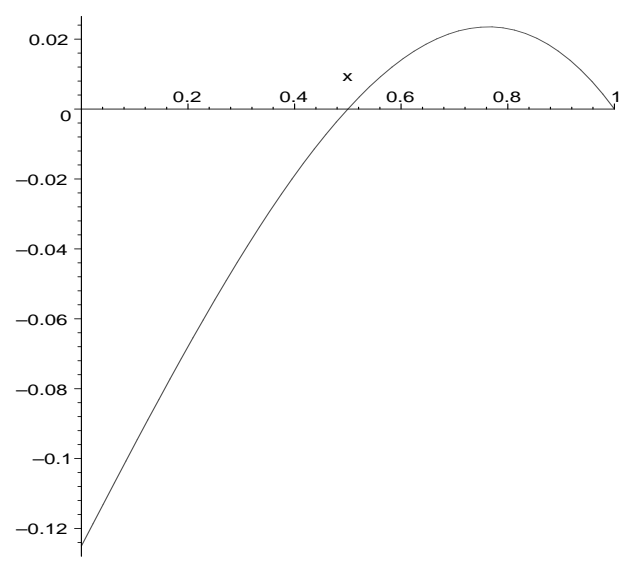

Figure 2. The solution $u(x)=-\frac{1}{6} x^{3}+\frac{7}{24} x-\frac{1}{8}$ computed by the hybrid, Lagrange and symmetric Baumann methods, $\sigma= \pm 1$

The computed Lagrange multipliers for the hybrid and Lagrange formulation are shown in Table 1. We see that in the case of the hybrid formulation, the Lagrange multipliers correspond with the fluxes at the boundaries, i.e., $p_{h}(0)=\frac{d u}{d n}(0)$ and $p_{h}(1)=\frac{d u}{d n}(1)$, whereas for the Lagrange formulation the Lagrange multipliers vanish.

Table 1. The values of the Lagrange multipliers of the hybrid and Lagrange methods for the solution as in Fig. 2

\begin{tabular}{|ll|l|l|}
\hline Lagrange method & $(3.2)$ & $p_{h}(d)=0$ & $p_{h}(1)=0$ \\
\hline hybrid method & $(3.3)$ & $p_{h}(0)=-5 / 25$ & $p_{h}(1)=-7 / 24$ \\
\hline
\end{tabular}

Next we check if we can solve (2.1) for an arbitrary location $d \in[0,1)$ of the interior Dirichlet boundary condition. Now we see that the dependencies on $d$ and $\sigma$ differ for the three methods. In the case of the symmetric- or Baumann-Oden method, we have to solve a full $(p+1) \times(p+1)$ linear system $L_{\sigma, d} u_{h}=f_{h}$, where the matrix depends on both the method parameter $\sigma$ and the interior boundary location $d$. 
In contrast, if we consider the coefficients of the linear system arising from the hybrid and the Lagrange methods, we observe the following block-partitioning:

$$
L_{d} u_{h}=\left(\begin{array}{ll}
\mathbf{A} & \mathbf{B} \\
\mathbf{C} & \mathbf{0}
\end{array}\right) u_{h}=f_{h}
$$

where, for the Lagrange method, $\mathbf{A} \cong \int_{0}^{1} u_{h}^{\prime} v_{h}^{\prime} d x-\left[u_{h}^{\prime}(1,0) v_{h}(1,0)\right]$ is the $(p+1) \times(p+1)$ leading submatrix, and $\mathbf{B} \cong\left[\bar{p}_{h}(d, 1) v_{h}(d, 1)\right]$ and $\mathbf{C} \cong\left[q_{h}(d, 1) u_{h}(d, 1)\right]$ have, respectively, dimensions $(p+1) \times 2$ and $2 \times(p+1)$. The dependence on $d$ is reflected in the elements of $\mathbf{B}$ and $\mathbf{C}$.

On the other hand, in the case of the hybrid-method, we have a $(p+1) \times(p+1)$ leading submatrix $\mathbf{A} \cong \int_{0}^{1} u_{h}^{\prime} v_{h}^{\prime} d x$. Now the $(p+1) \times 2$ submatrix $\mathbf{B} \cong\left[p_{h}(0,1) v_{h}(0,1)\right]$ is independent of $d$. The dependence on $d$ is only reflected in the $2 \times(p+1)$ matrix $\mathbf{C} \cong\left[q_{h}(d, 1) u_{h}(d, 1)\right]$.

Thus, we check if there are locations $d \in[0,1)$ in which any of the three methods may become singular. The results are shown in Table 2. We see that both the Lagrange and the symmetric Baumann methods have interior boundary locations where the methods become singular. The number of points where a singularity appears increases with the degree of polynomial. The hybrid method, however, shows no singular points. This motivates us to continue mainly with the hybrid method for the two-dimensional numerical experiments.

Table 2. Values of $d$ for which the discrete system becomes singular.

\begin{tabular}{|c|c|c|c|c|c|}
\hline$p$ & \multicolumn{3}{|c|}{ The Lagrange method } & \multicolumn{2}{c|}{ The symmetric Baumann method } \\
\hline 2 & $1 / 3$ & - & - & - & - \\
\hline 3 & $2 / 5-1 / 10 \sqrt{6}$ & $2 / 5+1 / 10 \sqrt{6}$ & - & $2 / 5$ & - \\
\hline 4 & 0.08858795951 & 0.4094668644 & 0.7876594618 & $3 / 7-1 / 7 \sqrt{2}$ & $3 / 7+1 / 7 \sqrt{2}$ \\
\hline
\end{tabular}

The discretizations are made for $S_{h}(\widehat{\Omega})=P^{p}(0,1), p=2,3,4$.

\subsection{Numerical experiments for the hybrid method on two-dimensional problems}

Having studied the one-dimensional discretization for various weak formulations with an embedded Dirichlet boundary condition, we now consider the two-dimensional Poisson equation on the unit square $\widehat{\Omega}$ as in (2.1) with an embedded Dirichlet boundary condition on a line parallel to the diagonal. For this line we use the following parametrization (see Fig. 3):

$$
\left\{\begin{array} { l l } 
{ x ( s ) = 1 - d / \sqrt { 2 } + s , } \\
{ y ( s ) = 1 - d / \sqrt { 2 } - s , }
\end{array} \quad \text { with } \left\{\begin{array}{ll}
|s|<d / \sqrt{2} & \text { if } 0 \leqslant d \leqslant 1 / \sqrt{2} \\
|s|<1-d / \sqrt{2} & \text { if } 1 / \sqrt{2}<d<\sqrt{2}
\end{array}\right.\right.
$$

Approximation with piecewise quadratics. To discretize the hybrid formulation, we first introduce the quadratic polynomial basis on the unit interval

$$
P^{2}([0,1])=\operatorname{Span}\{1-t, t, t(1-t)\} .
$$



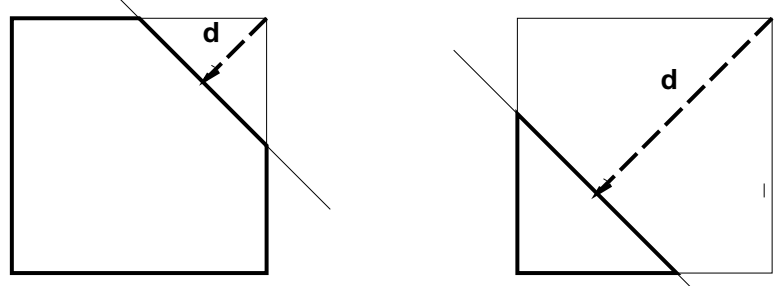

Figure 3. The domain $\Omega$ and its parametrization

We provide the test and trial function spaces with the 9-dimensional subspace $S_{h}(\widehat{\Omega})=$ $P^{2 \times 2}(\widehat{\Omega})=P^{2}(x) \otimes P^{2}(y) \subset H^{1}(\widehat{\Omega})$, i.e., the tensor product set of polynomials of degree $\leqslant 2$ in the two coordinate directions. Since we know that the Lagrange multiplier of the hybrid method corresponds with the flux $p=\mathbf{n} \cdot \nabla u$ on the boundary $\partial \widehat{\Omega}$, we choose to discretize the Lagrange multiplier as $p_{h}=\left.n_{x} \psi_{x}(x, y)\right|_{\partial \widehat{\Omega}}+\left.n_{y} \psi_{y}(x, y)\right|_{\partial \widehat{\Omega}}$, with $\psi \in P^{2 \times 2}(\widehat{\Omega})$ which defines the polynomial subspace $Q_{h}(\partial \widehat{\Omega}) \subset H^{-1 / 2}(\partial \widehat{\Omega})$ and also $Q_{h}(\partial \Omega) \subset H^{-1 / 2}(\partial \Omega)$ by $\left.p_{h}\right|_{\partial \Omega}=\left.n_{x} \psi_{x}(x, y)\right|_{\partial \Omega}+\left.n_{y} \psi_{y}(x, y)\right|_{\partial \Omega}$. Then the discrete formulation of the hybrid form is: find $u_{h} \in S_{h}(\widehat{\Omega}), p_{h} \in Q_{h}(\partial \widehat{\Omega})$ such that

$$
\begin{aligned}
\left\langle\nabla u_{h}, \nabla v_{h}\right\rangle_{S_{h}(\widehat{\Omega})}-\left\langle p_{h}, v_{h}\right\rangle_{Q_{h}(\partial \widehat{\Omega})} & =\left\langle f, v_{h}\right\rangle_{S_{h}(\widehat{\Omega})} \quad \forall v_{h} \in S_{h}(\widehat{\Omega}), \\
\left\langle q_{h}, u_{h}\right\rangle_{Q_{h}(\partial \Omega)} & =\left\langle q, u_{0}\right\rangle_{Q_{h}(\partial \Omega)} \quad \forall q \in Q_{h}(\partial \Omega),
\end{aligned}
$$

where the approximations are given by (9 degrees of freedom describe the polynomial in the interior)

$$
u_{h}(x, y)=\sum_{0 \leqslant i \leqslant 8} c_{i} \phi_{i}(x, y), \quad \phi_{i} \in S_{h}(\widehat{\Omega}),(x, y) \in \widehat{\Omega},
$$

and (note that 8 degrees of freedom describe the quadratic polynomials at the 4 boundaries)

$$
\begin{aligned}
p_{h}(x, y)= & \sum_{0 \leqslant i \leqslant 7} a_{i}\left[\left.n_{x} \psi_{x, i}(x, y)\right|_{\partial \widehat{\Omega}}+\left.n_{y} \psi_{y, i}(x, y)\right|_{\partial \widehat{\Omega}}\right] \\
& \left.\psi_{x, i}\right|_{\partial \widehat{\Omega}},\left.\psi_{y, i}\right|_{\partial \widehat{\Omega}} \in Q_{h}(\partial \widehat{\Omega}), \quad(x, y) \in \partial \widehat{\Omega} .
\end{aligned}
$$

The result is a $17 \times 17$ linear system depending on the diagonal distance of the embedded Dirichlet boundary to the origin. It is obvious that all methods will become ill-conditioned for values of $d$ close to $\sqrt{2}$, when the region $\Omega$ vanishes. In order to see how the singularity develops for the hybrid method (2.6), we plot the 17 singular values as a function of the diagonal distance $d$. The result is shown in Fig. 4. We see that, as in the one-dimensional experiment, also for this experiment, there are no values of $d$ for which the discretization matrix becomes singular. Furthermore, the method is not ill-conditioned for values of $d$ even larger than one. 


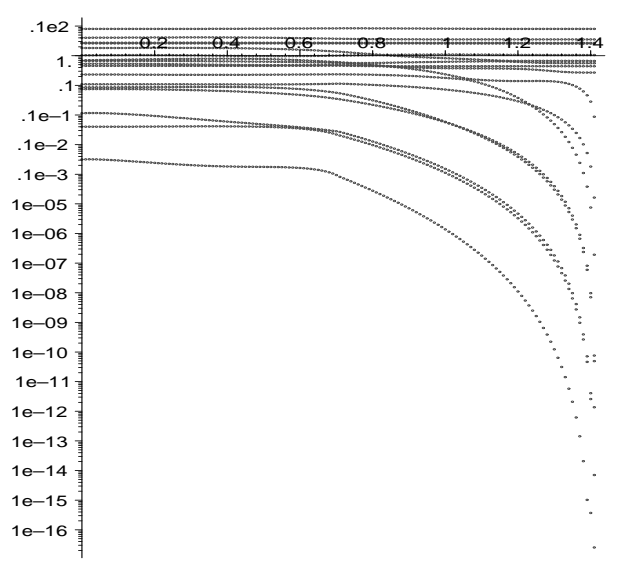

Figure 4. Singular values $\sigma_{i}, 1 \leqslant i \leqslant 17$ as a function of the diagonal distance $0 \leqslant d<\sqrt{2}$ for the third-order discretization of the hybrid method

Approximation with piecewise cubics. Next we want to study the stability of a higherorder discretization of the hybrid method (2.6) for the same two-dimensional model problem. To this end, we consider on the unit interval the cubic polynomial basis

$$
P^{3}([0,1])=\left\{1-t, t, t(1-t)^{2}, t^{2}(1-t)\right\}
$$

and we choose for the test- and trial function spaces the 16-dimensional subspace $S_{h}(\widehat{\Omega})=$ $P^{3 \times 3}(\widehat{\Omega})=P^{3}(x) \otimes P^{3}(y) \subset H^{1}(\widehat{\Omega})$, i.e., the tensor product polynomials of a degree less than four in the two coordinate directions. We choose the polynomial subspaces $Q_{h}(\partial \widehat{\Omega})=$ $\gamma_{1}^{\partial \widehat{\Omega}}\left(S_{h}(\widehat{\Omega})\right) \subset H^{-1 / 2}(\partial \widehat{\Omega})$ and $Q_{h}(\partial \Omega)=\gamma_{1}^{\partial \Omega}\left(S_{h}(\widehat{\Omega})\right) \subset H^{-1 / 2}(\partial \Omega)$. The choice of the basis functions in $Q_{h}(\partial \Omega)$ will be explained in the next section, where we study the general case with a curved boundary.

As explained in Section 4, using (3.5) and (3.6), we obtain a $28 \times 28$ system depending on the diagonal distance $d$ of the interior Dirichlet boundary to the origin. For this hybrid discretization, the 28 singular values as a function of $d$ are shown in Fig. 5. Generally, we observe the same behavior as for the quadratic polynomials.

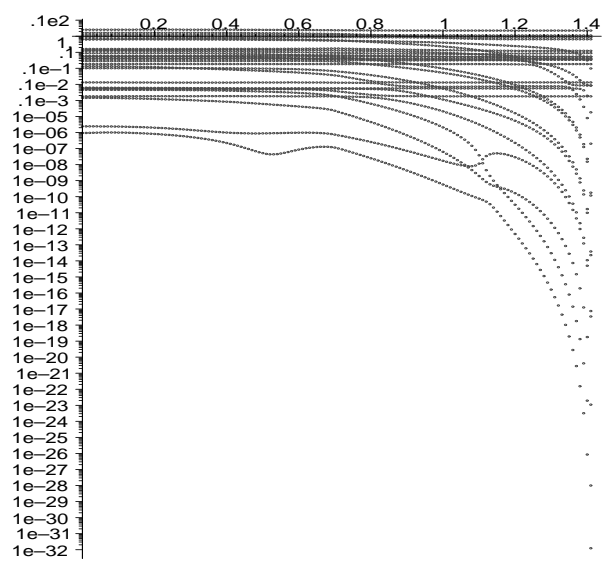

Figure 5. Singular values $\sigma_{i}, 1 \leqslant i \leqslant 28$ as a function of the diagonal distance $0 \leqslant d<\sqrt{2}$ for the fourth-order discretization of the hybrid method 


\section{Weak forms for embedded boundary conditions}

\subsection{The boundary condition on a curved embedded boundary}

In this section, we study the stability and accuracy of the fourth-order hybrid discretization of the Poisson equation on the unit square, with a part of the circular boundary embedded. So, we solve the equation

$$
-\Delta u=f \text { on } \widehat{\Omega} \quad \text { with } \quad u=u_{0} \text { on } \Gamma_{D}=\partial \Omega
$$

on the unit square from which a circle sector has been removed: i.e., $\widehat{\Omega}$ is the unit square and $\Gamma_{\text {int }} \subset \partial \Omega$, with $\Omega \subset \widehat{\Omega}$, is the circular curve

$$
\Gamma_{\mathrm{int}}=\left\{(x, y) \mid x^{2}+y^{2}=R^{2}<1, x \geqslant 0, y \geqslant 0\right\},
$$

and the fictitious part is

$$
\widetilde{\Omega}=\left\{(x, y) \mid x>0, y>0, x^{2}+y^{2}<R^{2}\right\} .
$$

The corresponding discrete hybrid formulation reads: find $u_{h} \in S_{h}(\widehat{\Omega}), \chi_{h} \in Q_{h}(\widehat{\Omega})$ such that

$$
\begin{array}{rlrl}
\left(\nabla u_{h}, \nabla v_{h}\right)_{\widehat{\Omega}}- & \left\langle\gamma_{1}^{\widehat{\Omega}}\left(\chi_{h}\right), \gamma_{0}^{\widehat{\Omega}}\left(v_{h}\right)\right\rangle_{\partial \widehat{\Omega}}=\left(f, v_{h}\right)_{\widehat{\Omega}}, & & \forall v_{h} \in S_{h}(\widehat{\Omega}), \\
& \left\langle\gamma_{1}^{\Omega}\left(q_{h}\right), \gamma_{0}^{\Omega}\left(u_{h}\right)\right\rangle_{\partial \Omega}=\left\langle\gamma_{1}^{\Omega}\left(q_{h}\right), u_{0}\right\rangle_{\partial \Omega}, & \forall q_{h} \in Q_{h}(\widehat{\Omega}),
\end{array}
$$

where $S_{h}(\widehat{\Omega}) \subset H^{1}(\widehat{\Omega})$ and $Q_{h}(\widehat{\Omega}) \subset H^{1}(\widehat{\Omega})$ are the proper finite dimensional polynomial subspaces, and $\gamma_{0}^{\Omega}, \gamma_{1}^{\Omega}$ and $\gamma_{0}^{\widehat{\Omega}}, \gamma_{1}^{\widehat{\Omega}}$ are the usual trace operators on $\partial \Omega$ and $\partial \widehat{\Omega}$, respectively. To provide these subspaces with a basis, we choose the cubic polynomials and consider the following polynomial basis on the unit interval:

$$
\phi_{1}=1-t, \quad \phi_{2}=(1-t)^{2} t, \quad \phi_{4}=t, \quad \phi_{3}=(1-t) t^{2} .
$$

We recognize that $\phi_{1}(t)$ and $\phi_{4}(t)$ are associated with the function values at $t=0,1$, respectively, while $\phi_{2}(t)$ and $\phi_{3}(t)$ can be associated with the corrections for the derivatives at $t=0,1$. These facts help us to understand the structure behind different polynomial subspaces that are constructed below.

First we choose for the test and trial function spaces a 16-dimensional subspace, i.e., $S_{h}(\widehat{\Omega})=P^{3 \times 3}(\widehat{\Omega})=P^{3}(x) \otimes P^{3}(y) \subset H^{1}(\widehat{\Omega})$, the usual tensor product of polynomials of a degree less than four in the two coordinate directions. Hence, on the unit square $\widehat{\Omega}$ we get the approximation $u_{h} \in S_{h}(\widehat{\Omega})$

$$
u_{h}=\sum_{1 \leqslant i, j \leqslant 4} c_{i, j} \phi_{i}(y) \phi_{j}(x) .
$$

Next we consider the usual trace operators, $\gamma_{0}^{\widehat{\Omega}}: H^{1}(\widehat{\Omega}) \rightarrow H^{1 / 2}(\partial \widehat{\Omega})$ and $\gamma_{0}^{\Omega}: H^{1}(\widehat{\Omega}) \rightarrow$ $H^{1 / 2}(\partial \Omega)$ applied to the boundary of $\widehat{\Omega}$ and $\Omega$ respectively, and, similarly, $\gamma_{1}^{\widehat{\Omega}}: H^{1}(\widehat{\Omega}) \rightarrow$ $H^{-1 / 2}(\partial \widehat{\Omega})$ and $\gamma_{1}^{\Omega}: H^{1}(\widehat{\Omega}) \rightarrow H^{-1 / 2}(\partial \Omega)$ the traces for the normal derivatives. We see that the approximating space of tensor product cubics, $S_{h}(\widehat{\Omega}) \subset H^{1}(\widehat{\Omega})$, is a 16-dimensional subspace. The trace of this space on $\partial \widehat{\Omega}$, the space $\gamma_{0}^{\widehat{\Omega}}\left(S_{h}(\widehat{\Omega})\right)$, however, is 12-dimensional, because the trace consists of independent cubics on the four edges, related by four continuity 
conditions at the vertices. Choosing the polynomial basis (4.3), in $S_{h}(\widehat{\Omega})$ the basis for $\gamma_{0}^{\widehat{\Omega}}\left(S_{h}(\widehat{\Omega})\right)$ can readily be found as a subset of the tensor product of the basis functions (4.3), by splitting $S_{h}(\widehat{\Omega})$ into two linearly independent subspaces:

$$
S_{h}(\widehat{\Omega})=\widetilde{Q}_{h}(\widehat{\Omega}) \oplus \widetilde{K}_{h}(\widehat{\Omega})
$$

with

$$
\widetilde{K}_{h}(\widehat{\Omega})=\operatorname{ker}\left(\gamma_{0}^{\widehat{\Omega}}\right) \cap S_{h}(\widehat{\Omega})=\operatorname{Span}\left(\phi_{i}(x) \phi_{j}(y) \mid i, j=2,3\right)
$$

and

$$
\widetilde{Q}_{h}(\widehat{\Omega})=\operatorname{Span}\left(\phi_{1}(x) \phi_{j}(y), \phi_{4}(x) \phi_{j}(y), \phi_{i}(x) \phi_{1}(y), \phi_{i}(x) \phi_{4}(y) ; i, j=1,2,3,4\right) .
$$

For the approximating space for $\gamma_{0}^{\widehat{\Omega}}\left(H^{1}(\widehat{\Omega})\right)$ we take

$$
Q_{h}^{*}(\partial \widehat{\Omega})=\gamma_{0}^{\widehat{\Omega}}\left(S_{h}(\widehat{\Omega})\right)=\gamma_{0}^{\widehat{\Omega}}\left(\widetilde{Q}_{h}(\widehat{\Omega})\right) \subset H^{1 / 2}(\partial \widehat{\Omega}) .
$$

Similarly, we introduce the approximation space for the traces on $\partial \Omega$ as

$$
Q_{h}^{*}(\partial \Omega)=\gamma_{0}^{\Omega}\left(\widetilde{Q}_{h}(\widehat{\Omega})\right) \subset H^{1 / 2}(\partial \Omega) .
$$

On the other hand, for the approximation of the trace of the normal derivatives we split the space $S_{h}(\widehat{\Omega})$ as

$$
S_{h}(\widehat{\Omega})=Q_{h}(\widehat{\Omega}) \oplus K_{h}(\widehat{\Omega})
$$

with

$$
K_{h}(\widehat{\Omega})=\operatorname{ker}\left(\gamma_{1}^{\widehat{\Omega}}\right) \cap S_{h}(\widehat{\Omega})=\operatorname{Span}\left(\psi_{i}(x) \psi_{j}(y) \mid i, j=1,4\right) \text { with } \psi_{k}=\phi_{k}-\phi_{3}-\phi_{2},
$$

and

$$
Q_{h}(\widehat{\Omega})=\operatorname{Span}\left(\phi_{2}(x) \phi_{j}(y), \phi_{3}(x) \phi_{j}(y), \phi_{i}(x) \phi_{2}(y), \phi_{i}(x) \phi_{3}(y), i, j=1,2,3,4\right) .
$$

We see that $Q_{h}(\widehat{\Omega})$ is 12-dimensional and $K_{h}(\widehat{\Omega})$ is 4-dimensional. The normal derivatives on the four edges of $\widehat{\Omega}$ are all approximated by cubic polynomials related by the condition that at the vertices $\frac{1}{\partial x}\left(\frac{\partial u_{h}}{\partial y}\right)=\frac{1}{\partial y}\left(\frac{\partial u_{h}}{\partial x}\right)$. So we find the approximating space for the normal derivatives at the boundary of $\widehat{\Omega}$, viz.,

$$
Q_{h}(\partial \widehat{\Omega})=\gamma_{1}^{\widehat{\Omega}}\left(S_{h}(\widehat{\Omega})\right)=\gamma_{1}^{\widehat{\Omega}}\left(Q_{h}(\widehat{\Omega})\right) \subset H^{-1 / 2}(\partial \widehat{\Omega})
$$

and at the boundary of $\Omega$ as

$$
Q_{h}(\partial \Omega)=\gamma_{1}^{\Omega}\left(Q_{h}(\widehat{\Omega})\right) \subset H^{-1 / 2}(\partial \Omega)
$$

Considering the Lagrange multiplier function $p \in H^{-1 / 2}(\partial \widehat{\Omega})$ in $(2.6)$, we know that if $u$ satisfies the Poisson equation (4.1) and also the Dirichlet boundary condition, the Lagrange multiplier $p$ on $\partial \widehat{\Omega}$ represents the normal flux $\mathbf{n} \cdot \nabla u$ at the boundary $\partial \widehat{\Omega}$, i.e., $p=\mathbf{n} \cdot \nabla u$. Thus, in the discrete hybrid formulation (4.2), we write for the Lagrange multiplier $p_{h}=$ 
$\mathbf{n} \cdot \nabla \chi_{h}$ on $\partial \widehat{\Omega}$, where $n$ is the unit outward normal vector and $\chi_{h} \in Q_{h}(\widehat{\Omega})$ is the master flux function given by

$$
\chi_{h}=\sum_{1 \leqslant i, j \leqslant 4} a_{i, j} \phi_{i}(x) \phi_{j}(y) \quad \text { with } \quad a_{i, j}=0, \quad i, j=1,4
$$

So we recognize the discrete hybrid formulation (4.2): find $u_{h} \in S_{h}(\widehat{\Omega}), \chi_{h} \in Q_{h}(\widehat{\Omega})$ such that

$$
\begin{array}{rlrl}
\left(\nabla u_{h}, \nabla v_{h}\right)_{\widehat{\Omega}}- & \left\langle\gamma_{1}^{\widehat{\Omega}}\left(\chi_{h}\right), \gamma_{0}^{\widehat{\Omega}}\left(v_{h}\right)\right\rangle_{\partial \widehat{\Omega}}=\left(f, v_{h}\right)_{\widehat{\Omega}} & & \forall v_{h} \in S_{h}(\widehat{\Omega}), \\
& \left\langle\gamma_{1}^{\Omega}\left(q_{h}\right), \gamma_{0}^{\Omega}\left(u_{h}\right)\right\rangle_{\partial \Omega}=\left\langle\gamma_{1}^{\Omega}\left(q_{h}\right), u_{0}\right\rangle_{\partial \Omega} & \forall q_{h} \in Q_{h}(\widehat{\Omega}),
\end{array}
$$

as a $(16+12) \times(16+12)$ linear system.

To study the stability of this hybrid formulation, we plot the singular values of the discrete $28 \times 28$ system as a function of the circle radius, $0<R \leqslant \sqrt{2}$. The result is shown in Fig. 6. In this figure we see 28 singular values as a function of the circle radius, $R$. The discrete formulation is sufficiently stable up to circle radii of $R \approx 1.1$. In this case, more than $80 \%$ of the total domain $\widehat{\Omega}$ consists of the fictitious domain $\widetilde{\Omega}$. The reason for the cusps in the figure near $R=0.4$ and $R=0.9$ is unknown.

Next, we check how the cubic approximation will be solved for the exact solution by taking in (4.1) the right-hand side and the boundary conditions such that the solution is given by $u=x^{3}+y^{3}+x y$. The solution and the error for two possible domains $(R=2 / 5$ and $R=4 / 5$ ) are shown in the Figs. 7 and 8 . We see that the hybrid formulation finds the exact solution on the domain $\Omega$, except for the rounding errors corresponding to the condition of the linear system.

To check the approximation behavior of the method we repeat the experiment for the solution $u(x, y)=e^{x+y}$ in (4.1). The solution and the error for both domains $(R=2 / 5$ and $R=4 / 5)$ are shown in the Figs. 9 and 10 .

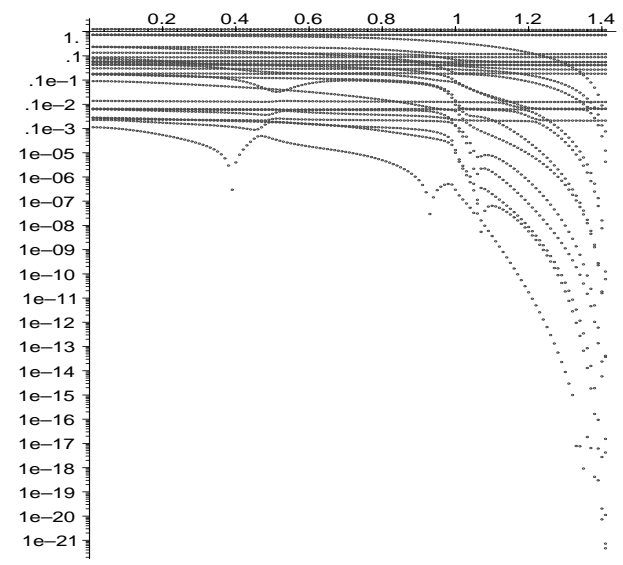

Figure 6. Singular values as a function of the embedded circle bow radius for the fourth-order hybrid discretization. On the fictitious part of the domain, the solution and the error are set equal to zero 

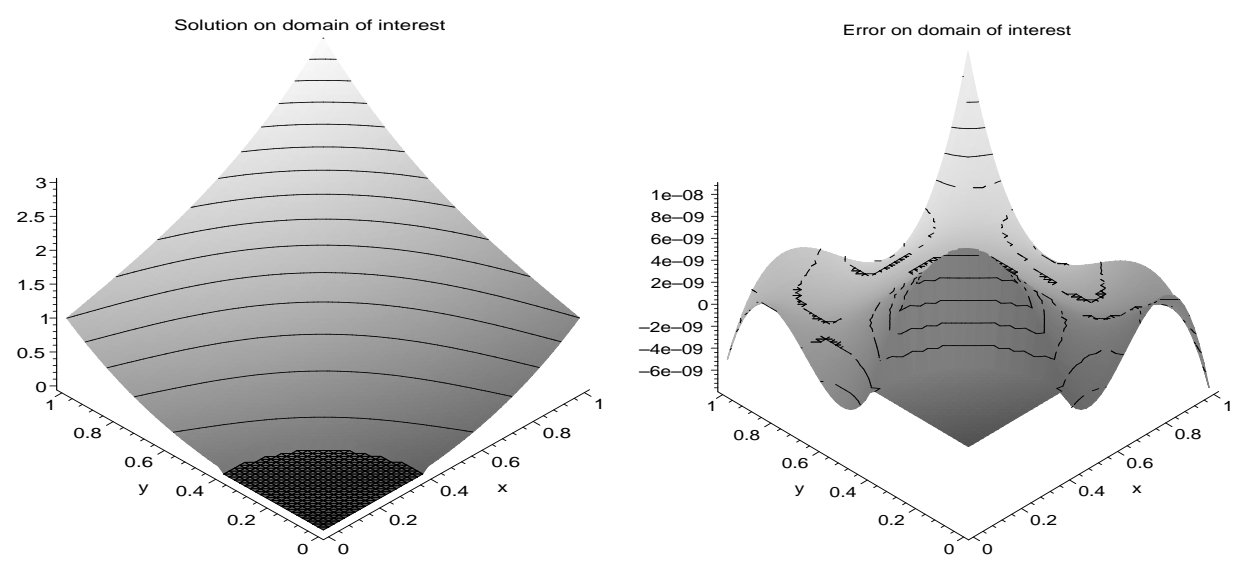

Figure 7. The solution $u=x^{3}+y^{3}+x y$ and the error on the domain $\Omega$ of the fourth-order hybrid discretization $(R=2 / 5)$
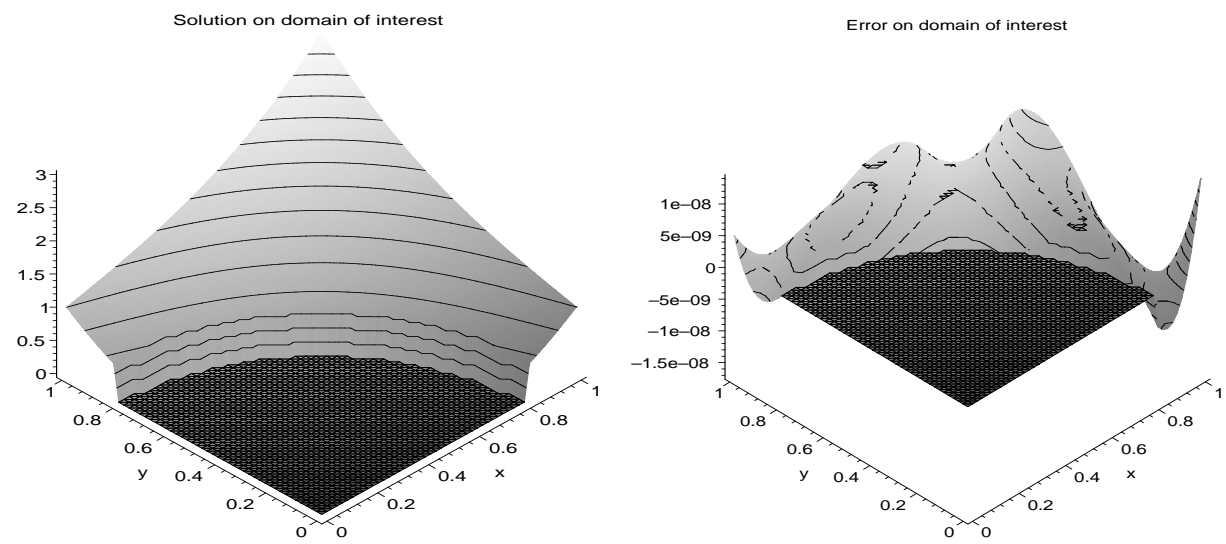

Figure 8. The solution $u=x^{3}+y^{3}+x y$ and the error on the domain $\Omega$ of the fourth-order hybrid discretization $(R=4 / 5)$
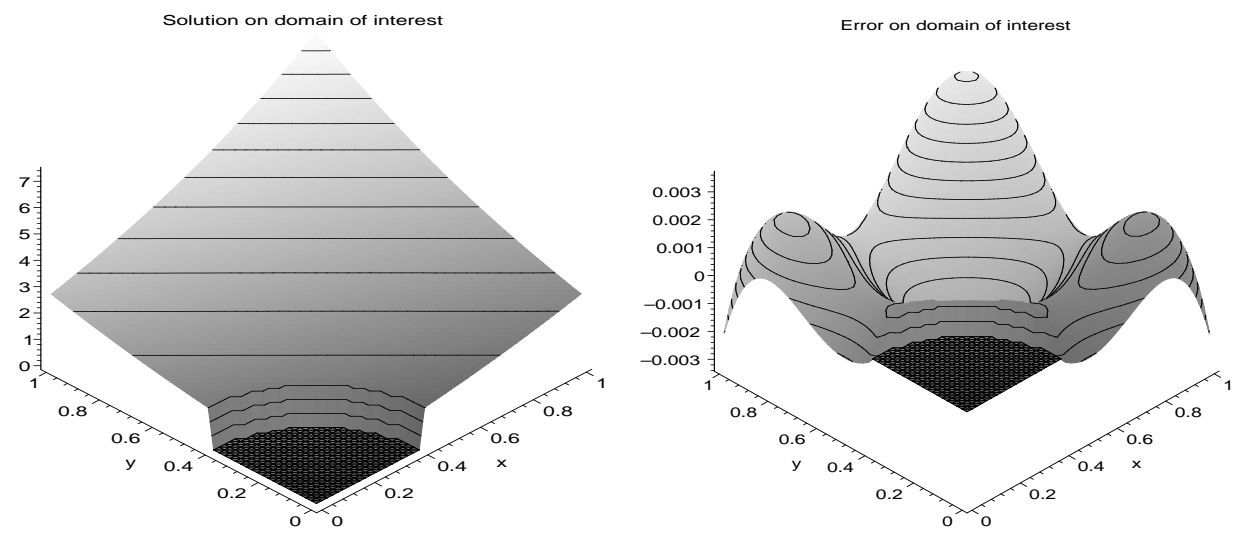

Figure 9. The solution $u=e^{x+y}$ and the error on the domain $\Omega$ of the fourth-order hybrid discretization $(R=2 / 5)$ 

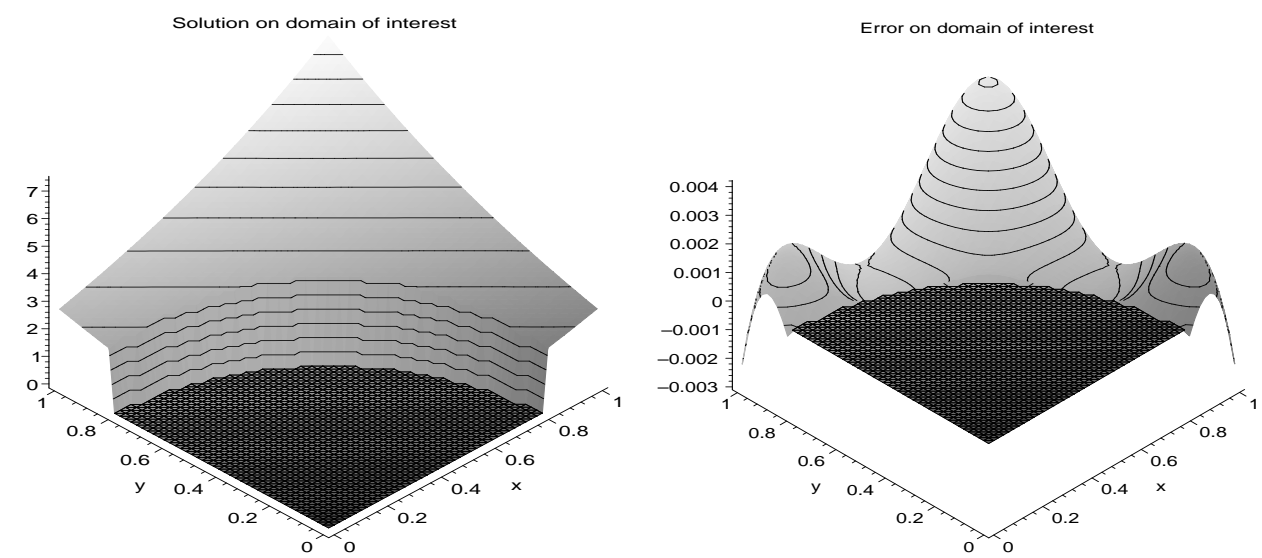

Figure 10. The solution $u=e^{x+y}$ and the error on the domain $\Omega$ of the fourth-order hybrid discretization $(R=4 / 5)$

\subsection{The combination of the hybrid and the discontinuous Galerkin formulation}

In the previous sections we have seen that the hybrid form is stable on a cell with an embedded Dirichlet boundary condition, whereas the discontinuous Galerkin method is not always stable. On the other hand, the discontinuous Galerkin method is cheaper, because the Lagrange multiplier has been eliminated and hence less degrees of freedom are involved. So, to reduce computational costs, if we consider a large regular rectangular grid on which, locally, there exist cells with embedded Dirichlet boundary conditions, it is natural to treat these cells with the hybrid method for stability, while the 'normal' rectangular cells are treated with the DG-Galerkin discretization.

To study such a method, we consider two adjacent rectangular cells $\widehat{\Omega}_{1}$ and $\widehat{\Omega}_{2}$, where only $\widehat{\Omega}_{1}$ has an embedded Dirichlet boundary condition. The cells have a common interface $\Gamma_{1,2}$. Because the cell $\widehat{\Omega}_{1}$ has an embedded boundary condition, we treat this cell with a hybrid discretization. The cell $\widehat{\Omega}_{2}$ is discretized by the DG discretization. So, on the cell $\widehat{\Omega}_{1}$ we have

$$
\begin{aligned}
\int_{\widehat{\Omega}_{1}} \nabla u_{h} \cdot \nabla u_{h} d x & -\int_{\Gamma_{1,2}}\left(\mathbf{n}_{1,2} \cdot \nabla \chi_{h}\right) v_{h} d s-\int_{\partial \widehat{\Omega}_{1} \backslash \Gamma_{1,2}}\left(\mathbf{n} \cdot \nabla \chi_{h}\right) v_{h} d s-\int_{\Gamma_{1,2}}\left(\mathbf{n}_{1,2} \cdot \nabla q_{h}\right) u_{h} d s \\
& -\int_{\partial \Omega_{1} \backslash \Gamma_{1,2}}\left(\mathbf{n} \cdot \nabla q_{h}\right) u_{h} d s=\int_{\widehat{\Omega}_{1}} f v_{h} d x-\int_{\partial \Omega_{1} \backslash \Gamma_{1,2}} \mathbf{n} \cdot \nabla q_{h} u_{0} d s,
\end{aligned}
$$

where $\mathbf{n}$ is the unit normal on the interface $\Gamma_{1,2}$ pointing from the cell $\widehat{\Omega}_{1}$ towards the cell $\widehat{\Omega}_{2}$. On the other hand, on the cell $\widehat{\Omega}_{2}$ we consider (for simplicity) the symmetric DG discretization. Hence

$$
\begin{gathered}
\int_{\widehat{\Omega}_{2}} \nabla u_{h} \cdot \nabla u_{h} d x-\int_{\Gamma_{1,2}}\left(\mathbf{n} \cdot \nabla u_{h}\right) v_{h} d s-\int_{\partial \widehat{\Omega}_{2} \backslash \Gamma_{1,2}}\left(\mathbf{n} \cdot \nabla u_{h}\right) v_{h} d s-\int_{\Gamma_{2,1}}\left(\mathbf{n} \cdot \nabla v_{h}\right) u_{h} d s \\
-\int_{\partial \widehat{\Omega}_{2} \backslash \Gamma_{1,2}}\left(\mathbf{n} \cdot \nabla v_{h}\right) u_{h} d s=\int_{\widehat{\Omega}_{2}} f v d x-\int_{\partial \widehat{\Omega}_{2} \backslash \Gamma_{1,2}}\left(\mathbf{n} \cdot \nabla v_{h}\right) u_{0} d s .
\end{gathered}
$$

Now we have to couple the two cells at the interface $\Gamma_{1,2}$. Therefore, we have to satisfy the locality, consistency, and conservation conditions, as discussed in [1]. To meet these 
conditions, we define the average fluxes across the interface by

$$
\left\langle\widetilde{\nabla u_{h}}\right\rangle=\frac{1}{2}\left(\left.\nabla \chi_{h}\right|_{\partial \widehat{\Omega}_{1}}+\left.\nabla u_{h}\right|_{\partial \Omega_{2}}\right) \text { and }\left\langle\widetilde{\nabla v_{h}}\right\rangle=\frac{1}{2}\left(\left.\nabla q_{h}\right|_{\partial \widehat{\Omega}_{1}}+\left.\nabla v_{h}\right|_{\partial \Omega_{2}}\right),
$$

and the jumps by

$$
\left[u_{h}\right]=\left.u_{h}\right|_{\partial \widehat{\Omega}_{1}} \mathbf{n}_{1,2}+\left.u_{h}\right|_{\partial \widehat{\Omega}_{2}} \mathbf{n}_{2,1} .
$$

Then, combining (4.4) and (4.5), together with the flux and jump relations, we arrive at the form

$$
\begin{aligned}
\int_{\widehat{\Omega}_{1} \cup \widehat{\Omega}_{2}} \nabla u_{h} \cdot \nabla v_{h} d x & -\int_{\Gamma_{1,2}}\left\langle\widetilde{\nabla u_{h}}\right\rangle \cdot\left[v_{h}\right] d s-\int_{\Gamma_{1,2}}\left\langle\widetilde{\nabla v_{h}}\right\rangle \cdot\left[u_{h}\right] d s-\int_{\partial \widehat{\Omega}_{1} \backslash \Gamma_{1,2}}\left(\mathbf{n} \cdot \nabla \chi_{h}\right) v_{h} d s \\
& -\int_{\partial \widehat{\Omega}_{2} \backslash \Gamma_{1,2}}\left(\mathbf{n} \cdot \nabla u_{h}\right) v_{h} d s-\int_{\partial \Omega_{1} \backslash \Gamma_{1,2}}\left(\mathbf{n} \cdot \nabla q_{h}\right) u_{h} d s-\int_{\partial \widehat{\Omega}_{2} \backslash \Gamma_{1,2}}\left(\mathbf{n} \cdot \nabla v_{h}\right) u_{h} d s \\
& =\int_{\widehat{\Omega}_{1} \cup \widehat{\Omega}_{2}} f v_{h} d x-\int_{\partial \Omega_{1} \backslash \Gamma_{1,2}}\left(\mathbf{n} \cdot \nabla q_{h}\right) u_{0} d s-\int_{\partial \widehat{\Omega}_{2} \backslash \Gamma_{1,2}}\left(\mathbf{n} \cdot \nabla v_{h}\right) u_{0} d s .
\end{aligned}
$$

This weak form can immediately be used for discretization as described above. The solution and the error of such a combined discretization with cubic polynomials is shown in Fig. 11.
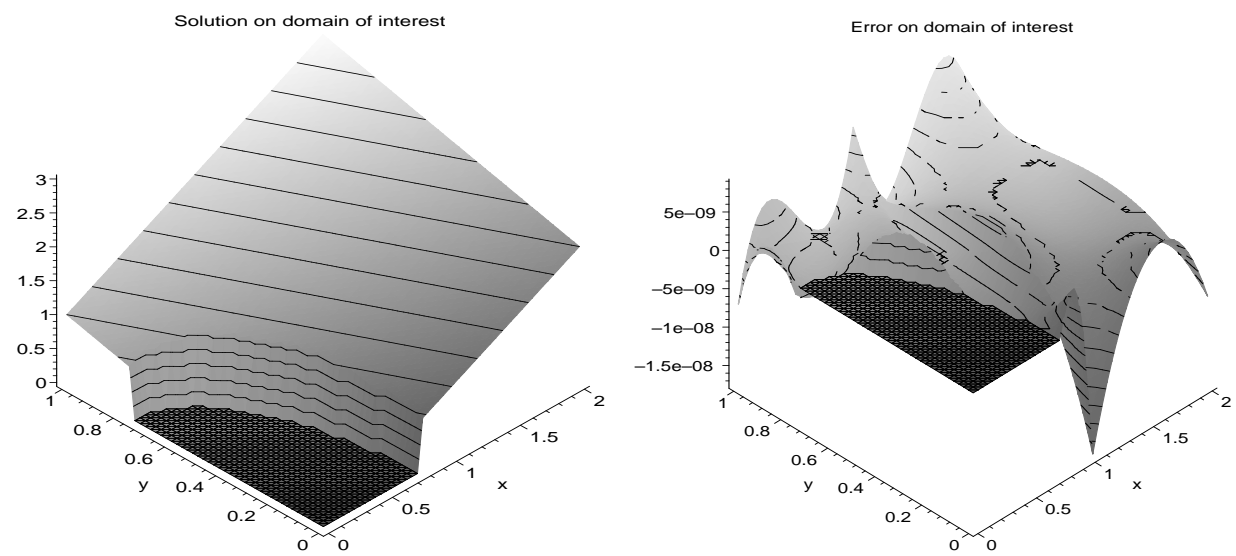

Figure 11. The approximate solution $u=x+y$ of $\Delta u=0$, and the error on the domain $\Omega_{1} \cup \widehat{\Omega}_{2}$ for a fourth-order combined hybrid-symmetric DG discretization with an embedded circle segment Dirichlet boundary condition $(R=3 / 4)$

\subsection{An embedded boundary for the convection equation}

Having studied the discretization of the Poisson equation, we now consider the convection equation with an interior Dirichlet boundary condition

$$
\mathbf{b} \cdot \nabla u=f \text { in } \widehat{\Omega}, \quad u=u_{0} \text { on } \partial \Omega_{\text {in }},
$$

where $\mathbf{b}$ is a constant vector denoting the direction of the convection and $\partial \Omega_{\text {in }}$ is the inflow boundary of $\Omega$ such that the boundary of $\Omega$ is $\partial \Omega=\partial \Omega_{\text {in }} \cup \partial \Omega_{\text {out }}$. The inflow and outflow boundaries are defined by $\mathbf{b} \cdot \mathbf{n}<0$ on $\partial \Omega_{\text {in }}$ and $\mathbf{b} \cdot \mathbf{n}>0$ on $\partial \Omega_{\text {out }}$, respectively. Considering 
the boundary of the whole domain $\partial \widehat{\Omega}$, we also split this boundary in an upwind and downwind boundary such that $\partial \widehat{\Omega}=\partial \widehat{\Omega}_{\text {in }} \cup \partial \widehat{\Omega}_{\text {out }}$. Then, according to the Lagrange multiplier theorem, we arrive, for the boundary value problem, at the following weak formulation: find $u \in H^{1}(\widehat{\Omega})$ and $\chi \in H^{1 / 2}\left(\partial \widehat{\Omega}_{\text {in }}\right)$ such that

$$
\begin{aligned}
-\int_{\widehat{\Omega}} \nabla v \cdot \mathbf{b} u d x & +\int_{\partial \widehat{\Omega}_{\mathrm{in}}} \mathbf{n} \cdot \mathbf{b} \chi v d s+\int_{\partial \widehat{\Omega}_{\mathrm{out}}} \mathbf{n} \cdot \mathbf{b} u v d s+\int_{\partial \Omega_{\mathrm{in}}} \mathbf{n} \cdot \mathbf{b} q u d s \\
& =\int_{\widehat{\Omega}} f v d x+\int_{\partial \Omega_{\mathrm{in}}} \mathbf{n} \cdot \mathbf{b} q u_{0} d s \quad \forall v \in H^{1}(\widehat{\Omega}), \forall q \in H^{1 / 2}\left(\partial \Omega_{\mathrm{in}}\right),
\end{aligned}
$$

in which we assume that $u$ on the fictitious domain $\widetilde{\Omega}$ satisfies the differential equation and is the continuation of the solution $u$ on the domain $\Omega$. Fig. 12 shows the solution and the error if (4.6) is used as the starting point for the discretization with cubic polynomials, as discussed above.
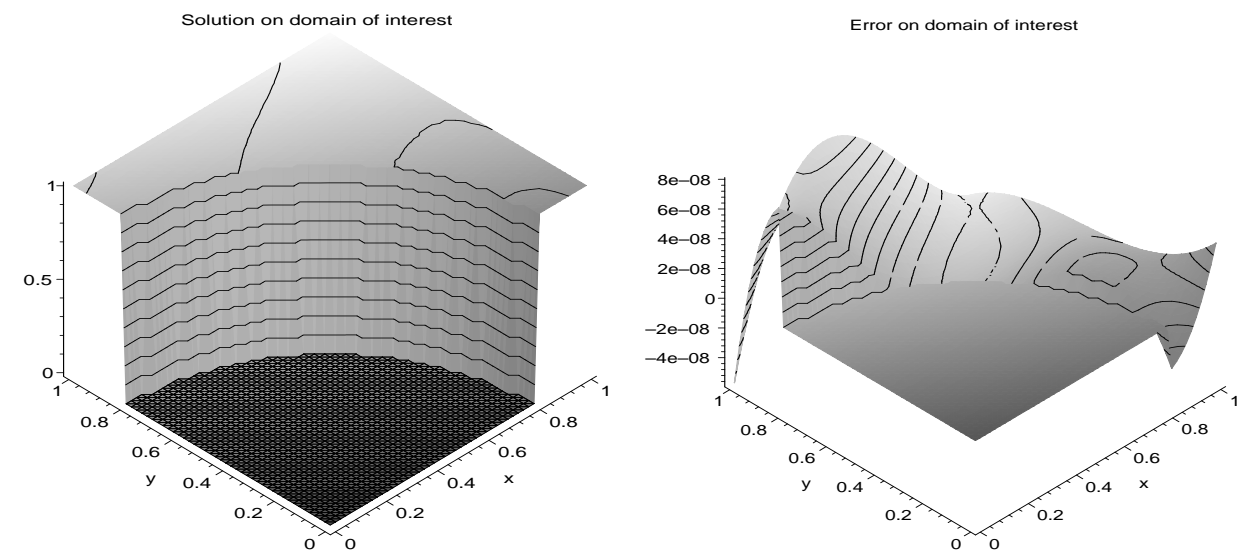

Figure 12. The approximate solution $u=1$ of the convection equation $\mathbf{b} \cdot \nabla u=0$ and the error on the domain $\Omega$ for the fourth-order hybrid discretization with an embedded circle segment Dirichlet boundary condition $(R=8 / 10)$

\subsection{Two adjacent cells with a common interior embedded boundary condition}

In this section, we study the finite element discretization of the convection diffusion equation

$$
-\varepsilon \Delta u+\mathbf{b} \cdot \nabla u=0
$$

discretized on two adjacent cells $\widehat{\Omega}_{1}$ and $\widehat{\Omega}_{2}$, with vertices $(-1,0),(0,0),(0,1),(-1,1)$ and $(0,0),(1,0),(1,1),(0,1)$, respectively. The embedded Dirichlet boundary condition is given on the half circle $x^{2}+y^{2}=R^{2}, 0 \leqslant y \leqslant 1$, so that the domain of interest is given by

$$
\Omega=\left(\widehat{\Omega}_{1} \cup \widehat{\Omega}_{2}\right) \backslash\left\{(x, y) \mid x^{2}+y^{2}<R^{2}\right\} .
$$

We first consider the diffusion part of the equation. Then the weak hybrid formulation of the problem reads: find $u \in H^{1}\left(\widehat{\Omega}_{h}\right)$ and $p \in H^{-1 / 2}\left(\partial \widehat{\Omega} \cup \widehat{\Gamma}_{\text {int }}\right)$ such that:

$$
\begin{aligned}
(\nabla u, \nabla v)_{\widehat{\Omega}_{h}} & -\langle p, v\rangle_{\partial \widehat{\Omega}}-\langle p, \mathbf{n} \cdot[v]\rangle_{\widehat{\Gamma}_{\mathrm{int}}}-\langle q, u\rangle_{\partial \Omega}-\langle q, \mathbf{n} \cdot[u]\rangle_{\Gamma_{\mathrm{int}}} \\
& =(f, v)_{\widehat{\Omega}_{h}}-\left\langle q, u_{0}\right\rangle_{\partial \Omega} \quad \forall v \in H^{1}\left(\widehat{\Omega}_{h}\right), q \in H^{-1 / 2}\left(\partial \Omega \cup \Gamma_{\mathrm{int}}\right),
\end{aligned}
$$


where $H^{1}\left(\widehat{\Omega}_{h}\right)$ is the broken Sobolev space on $\widehat{\Omega}_{1} \cup \widehat{\Omega}_{2}$ and the common interface is given by $\widehat{\Gamma}_{\text {int }}=\widehat{\Omega}_{1} \cap \widehat{\Omega}_{2}$, and $\mathbf{n}$ is the normal vector. The interface $\Gamma_{\text {int }}$, not including the fictitious part, is defined by $\Gamma_{\text {int }}=\bar{\Omega}_{1} \cap \bar{\Omega}_{2}$. Recognizing in $\left.p\right|_{\widehat{\Gamma}_{\text {int }}}$ a normal flux on the common interface $\widehat{\Gamma}_{\text {int }}$, we define the trace operators, $\widetilde{\gamma}_{1}^{\widehat{\Omega}}: H^{1}(\widehat{\Omega}) \cap C^{1}(\widehat{\Omega}) \rightarrow H^{-1 / 2}\left(\partial \widehat{\Omega} \cup \widehat{\Gamma}_{\text {int }}\right)$ and $\widetilde{\gamma}_{1}^{\Omega}: H^{1}(\widehat{\Omega}) \cap C^{1}(\widehat{\Omega}) \rightarrow H^{-1 / 2}\left(\partial \Omega \cup \Gamma_{\text {int }}\right)$. In order to approximate the normal derivatives on $\partial \widehat{\Omega} \cup \widehat{\Gamma}_{\text {int }}$, we proceed as in Section 4.1 and introduce the polynomial subspace $S_{h}(\widehat{\Omega}) \subset$ $H^{1}(\widehat{\Omega}) \cap C^{1}(\widehat{\Omega})$. We split this space as:

$$
S_{h}(\widehat{\Omega})=Q_{h}(\widehat{\Omega}) \oplus K_{h}(\widehat{\Omega})
$$

with

$$
K_{h}(\widehat{\Omega})=\operatorname{ker}\left(\widetilde{\gamma}_{1}^{\widehat{\Omega}}\right) \cap S_{h}(\widehat{\Omega})
$$

Then the discrete version of (4.8) reads: find $u_{h} \in S_{h}\left(\widehat{\Omega}_{h}\right)$ and $\chi_{h} \in Q_{h}(\widehat{\Omega})$ such that

$$
\begin{aligned}
\left(\nabla u_{h}, \nabla v_{h}\right)_{\widehat{\Omega}_{h}} & -\left\langle\widetilde{\gamma}_{1}^{\widehat{\Omega}}\left(\chi_{h}\right), \gamma_{0}\left(v_{h}\right)\right\rangle_{\partial \widehat{\Omega}}-\left\langle\widetilde{\gamma}_{1}^{\widehat{\Omega}}\left(\chi_{h}\right),\left[v_{h}\right]\right\rangle_{\widehat{\Gamma}_{\mathrm{int}}} \\
& -\left\langle\widetilde{\gamma}_{1}^{\Omega}\left(q_{h}\right), \gamma_{0}\left(u_{h}\right)\right\rangle_{\partial \Omega}-\left\langle\widetilde{\gamma}_{1}^{\Omega}\left(q_{h}\right),\left[u_{h}\right]\right\rangle_{\Gamma_{\mathrm{int}}} \\
& =\left(f, v_{h}\right)_{\widehat{\Omega}_{h}}-\left\langle\widetilde{\gamma}_{1}^{\Omega}\left(q_{h}\right), u_{0}\right\rangle_{\partial \Omega} \quad \forall v_{h} \in S_{h}\left(\widehat{\Omega}_{h}\right), q_{h} \in Q_{h}(\widehat{\Omega}) .
\end{aligned}
$$

(Note the polynomial spaces used!). For the polynomial space $S_{h}\left(\widehat{\Omega}_{h}\right)$ we can take the usual space of piecewise cubic polynomials in each coordinate direction on the partitioning $\widehat{\Omega}_{1} \cup \widehat{\Omega}_{2}$. On the other hand, it is not trivial to find a cubic polynomial space for $Q_{h}(\widehat{\Omega}) \subset$ $H^{1}(\widehat{\Omega}) \cap C^{1}(\widehat{\Omega})$. As we do not want to make our discretization unnecessarily complicated and expensive, we eliminate the extra degrees of freedom for $\chi_{h}$ by identifying them with $\nabla u_{h}$. Similarly, identifying $q_{h}$ and $\sigma \nabla v_{h}$ on $\partial \Omega \cup \Gamma_{\text {int }}$, we arrive at a discontinuous Galerkin discretization.

As our first interest is an efficient fourth-order discretization, we expect it to be highly improbable that instability will occur in the discrete operator, because the one-dimensional experiment shows only a single pole. Nevertheless, the use of the DG discretization forces us to monitor for possible singularities. Now the DG version of $(4.9)$ is: find $u_{h} \in S_{h}\left(\widehat{\Omega}_{h}\right)$ such that

$$
\begin{aligned}
\left(\nabla u_{h}, \nabla v_{h}\right)_{\widehat{\Omega}_{h}} & -\left\langle\nabla u_{h}, v_{h}\right\rangle_{\partial \widehat{\Omega}}-\left\langle\left\langle\nabla u_{h}\right\rangle,\left[v_{h}\right]\right\rangle_{\widehat{\Gamma}_{\mathrm{int}}}+\sigma\left\langle\nabla v_{h}, u_{h}\right\rangle_{\partial \Omega}+\sigma\left\langle\left\langle\nabla v_{h}\right\rangle,[u]\right\rangle_{\Gamma_{\mathrm{int}}} \\
= & \left(f, v_{h}\right)_{\widehat{\Omega}_{h}}+\sigma\left\langle\left\langle\nabla v_{h}\right\rangle, u_{0}\right\rangle_{\partial \Omega} \quad \forall v \in S_{h}\left(\widehat{\Omega}_{h}\right)
\end{aligned}
$$

with the usual choices for the normal flux functions.

Next we consider the convection part of (4.7). So, on the domain $\widehat{\Omega}$ we consider the equation

$$
\mathbf{b} \cdot \nabla u=f, \quad u=u_{0} \text { on } \partial \Omega_{\text {in }} .
$$

For simplicity, we set $\mathbf{b}=(1,0)$. Then the embedded boundary is an outflow boundary for $\widehat{\Omega}_{1}$, whereas for $\widehat{\Omega}_{2}$ it is an inflow boundary. Hence, we can neglect this embedded boundary in $\widehat{\Omega}_{1}$, whereas in cell the $\widehat{\Omega}_{2}$ we must introduce a Lagrange multiplier in order to satisfy the upwind boundary condition on the circle bow. Hence, we arrive at the following weak form 
for the convection part: find $u, \chi \in H^{1}\left(\widehat{\Omega}_{h}\right)=H^{1}\left(\widehat{\Omega}_{1} \cup \widehat{\Omega}_{2}\right)$

$$
\begin{aligned}
-\int_{\widehat{\Omega}} \nabla v \cdot \mathbf{b} u d x & +\int_{\partial \widehat{\Omega}_{1, \text { out }}}(\mathbf{n} \cdot \mathbf{b} u) v d s+\int_{\partial \widehat{\Omega}_{2, \text { in }}}(\mathbf{n} \cdot \mathbf{b} \chi) v d s+\int_{\partial \widehat{\Omega}_{2, \text { out }}}(\mathbf{n} \cdot \mathbf{b} u) v d s \\
& +\int_{\partial \Gamma_{\text {int }}}(\mathbf{n} \cdot \mathbf{b} q) u d s-\int_{\Gamma_{\mathrm{int}}}(\mathbf{n} \cdot \mathbf{b} q) u^{-} d s \\
= & \int_{\widehat{\Omega}} f v d x-\int_{\partial \widehat{\Omega}_{1, \text { in }}}\left(\mathbf{n} \cdot \mathbf{b} u_{0}\right) v d s+\int_{\Gamma_{D}}(\mathbf{n} \cdot \mathbf{b} q) u_{0} d s \quad \forall v, q \in H^{1}(\widehat{\Omega}),
\end{aligned}
$$

where $u^{-}=\left.u\right|_{\partial \Omega_{1}}=\left.u\right|_{\Gamma_{\text {int }}}$. If we want to eliminate in (4.11) the extra degrees of freedom, we set $\chi=u$ and $q=v$.
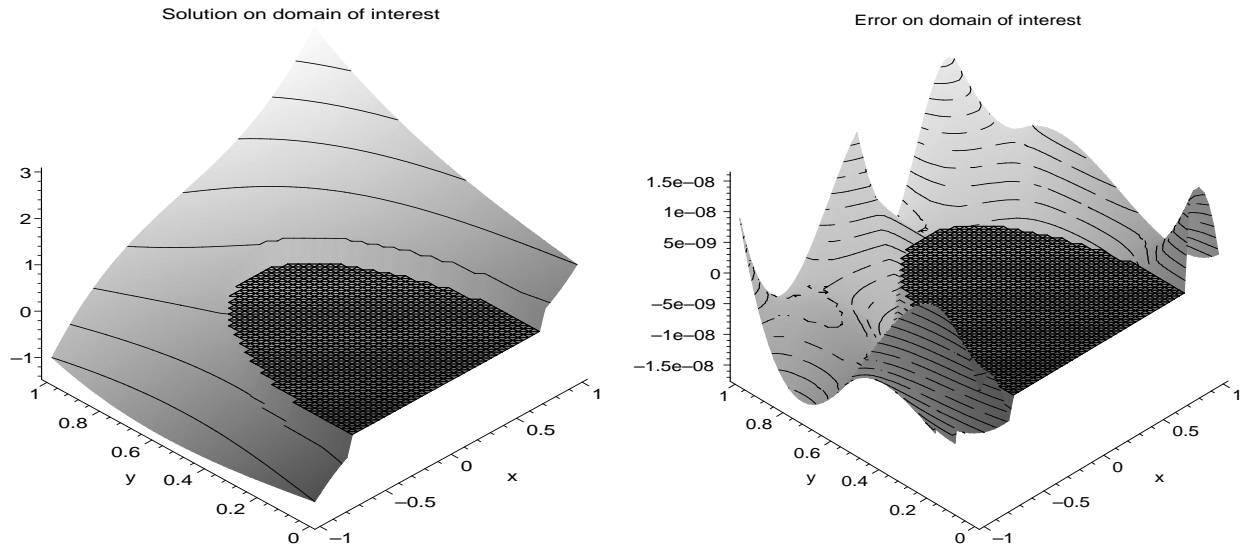

Figure 13. The approximate solution $u=x^{3}+y^{3}+x y$ of $-\Delta u+u_{x}=f$ and the error on the domain $\Omega=\Omega_{1} \cup \Omega_{2}$ for the fourth-order symmetric DG discretization with an embedded circle Dirichlet boundary condition $(R=3 / 4)$
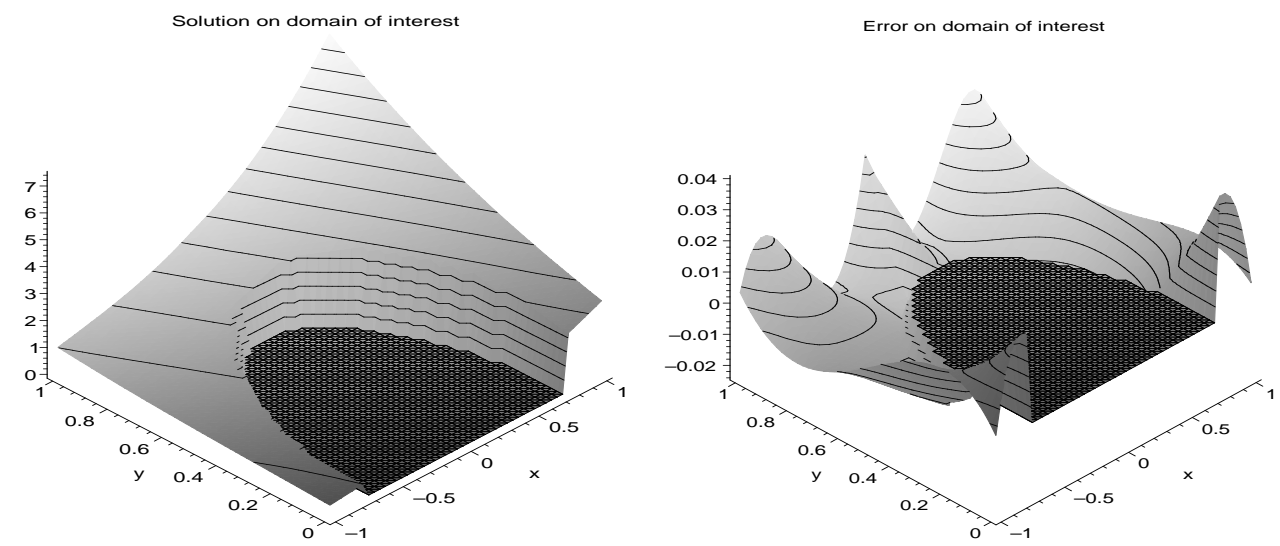

Figure 14. The approximate solution $u=e^{x+y}$ of $-\Delta u+u_{x}=f$ and the error on the domain $\Omega_{1} \cup \Omega_{2}$ for the fourth-order symmetric DG discretization with an embedded circle Dirichlet boundary condition $(R=3 / 4)$

The linear combination of (4.10) and (4.11) gives a discretization of the convection diffusion equation

$$
-\Delta u+\mathbf{b} \cdot \nabla u=f \text { in } \widehat{\Omega}, \quad u=u_{0} \text { on } \partial \Omega .
$$


Figs. 13 and 14 show the solution and the error of the discretization of (4.12) by means of (4.10) and (4.11) with tensor-product cubics as an approximation and test spaces.

\section{A singularly perturbed PDE on only two cells with a half circle excluded}

In this section, we are interested to solve the following convection diffusion problem (see Fig. 15). More details about this problem can be found in [5]

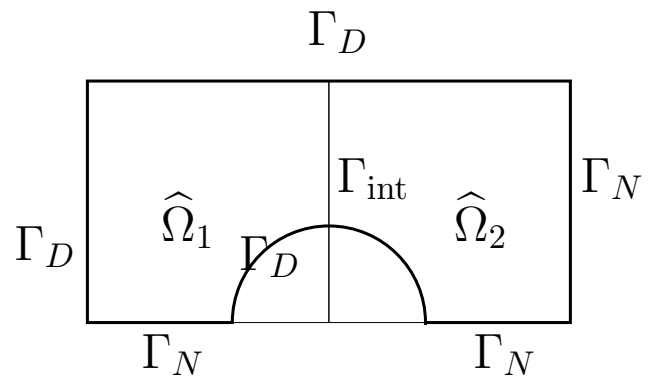

Figure 15. The domain for problem (5.1-5.2)

$$
\begin{aligned}
-\varepsilon \Delta u+u_{x}=f & \text { on } \widehat{\Omega}=\{(x, y) \mid-1<x<1,0<y<1\}, \\
u=0 & \text { on } \partial \Omega=\{(x, y) \mid x=-1,0<y<1 ;-1<x<1, y=1\}, \\
u=1 & \text { on } \Gamma_{D}=\left\{(x, y) \mid x^{2}+y^{2}=R^{2}, y>0 ; R<1\right\}, \\
\mathbf{n} \cdot \varepsilon \nabla u=0 & \text { on } \Gamma_{N}=\{(x, 0)|R<| x \mid<1\} \cup\{(1, y) \mid 0<y<1\} .
\end{aligned}
$$

Let $\widehat{\Omega}_{1}$ and $\widehat{\Omega}_{2}$ be two unit cells with respective vertices $(-1,0),(0,0),(0,1),(-1,1)$ and $(0,0),(1,0),(1,1),(0,1)$ so that $\overline{\widehat{\Omega}}=\widehat{\widehat{\Omega}}_{1} \cup \widehat{\widehat{\Omega}}_{2}$. For this problem, we want to study the symmetric and the Baumann-Oden DG-method.

First we study the diffusion part of (5.1) and replace the homogeneous Neumann boundary condition on $\Gamma_{N}=\{(x, y) \mid x=0,0<y<1\}$ with the homogeneous Dirichlet boundary condition in order to obtain a problem symmetric around $x=0$. Now the corresponding hybrid formulation (2.6) for (5.1) reads: find $u \in H^{1}\left(\widehat{\Omega}_{h}\right)$ and $p \in H^{-1 / 2}\left(\partial \widehat{\Omega} \cup \widehat{\Gamma}_{\text {int }}\right)$ such that

$$
\begin{aligned}
(\nabla u, \nabla v)_{\widehat{\Omega}} & -\langle p, v\rangle_{\partial \widehat{\Omega}}-\langle q, u\rangle_{\partial \Omega \cup \Gamma_{D}}-\langle p, \mathbf{n} \cdot[v]\rangle_{\widehat{\Gamma}_{\mathrm{int}}}-\langle q, \mathbf{n} \cdot[u]\rangle_{\Gamma_{\mathrm{int}}}= \\
& =(f, v)-\left\langle q, u_{0}\right\rangle_{\partial \Omega \cup \Gamma_{D}} \quad \forall v \in H^{1}\left(\widehat{\Omega}_{h}\right), q \in H^{-1 / 2}\left(\partial \widehat{\Omega} \cup \Gamma_{\text {int }}\right) .
\end{aligned}
$$

Here $H^{1}\left(\widehat{\Omega}_{h}\right)$ is a broken Sobolev space on $\widehat{\Omega}_{1} \cup \widehat{\Omega}_{2}$ and the jump operator is given by $[v]=\left.\mathbf{n}_{1} v\right|_{\partial \widehat{\Omega}_{1}}+\left.\mathbf{n}_{2} v\right|_{\partial \widehat{\Omega}_{2}}$. Further, $\Gamma_{\text {int }}=\partial \Omega_{1} \cap \partial \Omega_{2}$ is the interior wall on which the true solution is continuous. However, continuity is not required outside $\Omega$ and hence not on all $\widetilde{\Gamma}_{\text {int }}=\widehat{\Gamma}_{\text {int }} \cap \widetilde{\Omega}$.

To arrive at the DG-discretization of (5.3), we take for the test and trial space, $S_{h}(\widehat{\Omega}) \subset$ $H^{1}\left(\widehat{\Omega}_{h}\right)$, the tensor product of polynomials of degree $p<4$ in each of the coordinate directions 
and we write for the approximation

$$
u_{h}=\sum_{0 \leqslant e<2} \sum_{0 \leqslant i, j<4} c_{e, i, j} \phi_{e, i}(x) \phi_{e, j}(y) .
$$

In practice, we construct a basis from (4.3).

Next, for the DG discretization, we eliminate the extra equations and degrees of freedom for the Lagrange multiplier using the fact that $p$ represents the normal flux of $u$ at $\partial \widehat{\Omega}$ and at the internal wall $\widehat{\Gamma}_{\text {int }}$. Thus, replacing $p$ by $\mathbf{n} \cdot \nabla u_{h}$ on $\partial \widehat{\Omega}$ and by $\left\langle\mathbf{n} \cdot \nabla u_{h}\right\rangle$ on $\widehat{\Gamma}_{\text {int }}$ and replacing, similarly, $q$ by $-\sigma \mathbf{n} \cdot \nabla v$, we get the DG discretization of (5.3): find $u_{h} \in S_{h}(\widehat{\Omega})$ such that

$$
\begin{aligned}
\left(\nabla u_{h}, \nabla v_{h}\right)_{\widehat{\Omega}} & -\left\langle\mathbf{n} \cdot \nabla u_{h}, v_{h}\right\rangle_{\partial \widehat{\Omega}}+\sigma\left\langle\mathbf{n} \cdot \nabla v_{h}, u_{h}\right\rangle_{\partial \Omega \cup \Gamma_{D}}-\left\langle\left\langle\nabla u_{h}\right\rangle,[v]\right\rangle_{\widehat{\Gamma}_{\text {int }}} \\
& +\sigma\left\langle\left\langle\nabla v_{h}\right\rangle,[u]\right\rangle_{\Gamma_{\text {int }}}=\left(f, v_{h}\right)+\sigma\left\langle\mathbf{n} \cdot \nabla v_{h}, u_{0}\right\rangle_{\partial \Omega \cup \Gamma_{D}} \quad \forall v_{h} \in S_{h}(\widehat{\Omega}) .
\end{aligned}
$$

Solution on domain of interes

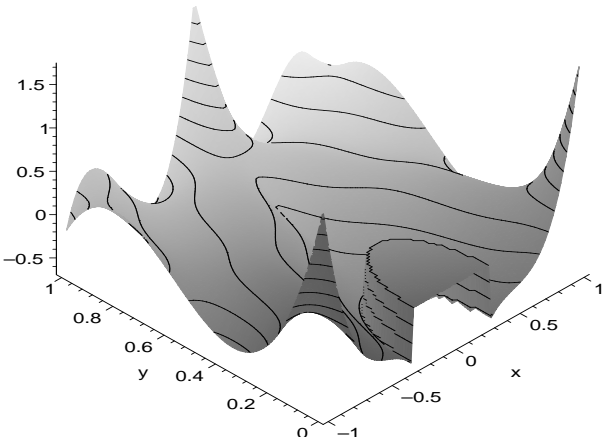

symmetric DG

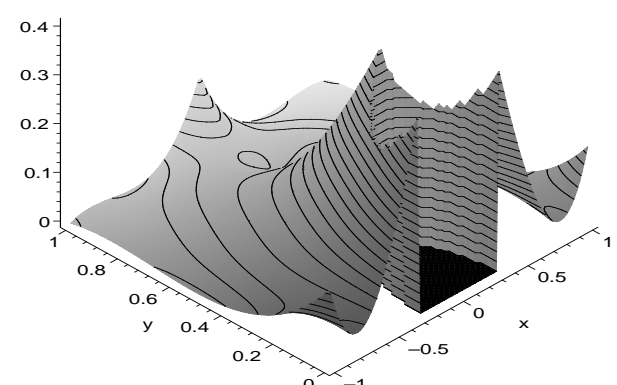

Baumann-Oden DG

Figure 16. The approximate solution $u_{h}$ of $\Delta u=0$ on the domain $\Omega$ with symmetric boundary conditions and fourth-order discretizations with an embedded circle Dirichlet boundary condition $(R=3 / 10)$

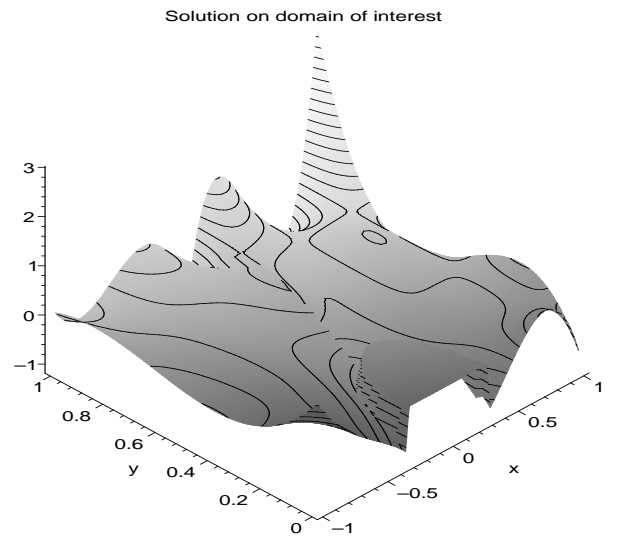

symmetric DG
Solution on domain of interes

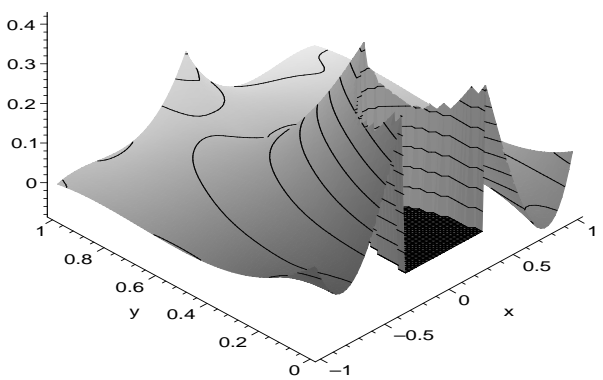

Baumann-Oden DG

Figure 17. The approximate solution $u_{h}$ of $\Delta u=0$ on the domain $\widehat{\Omega}$ with the boundary conditions (5.2) for a fourth-order symmetric and Baumann-Oden discontinuous Galerkin discretisation. The embedded circle Dirichlet boundary condition is located at $R=3 / 10$. 
Fig. 16 shows the solution of the symmetric $(\sigma=-1)$ and Baumann-Oden $(\sigma=1)$ discretization. We see that both solutions are symmetric, indeed, because of the symmetric structure of the problem. On the other hand, we recognize the unstable behavior of the symmetric DG method, which is of a poor quality compared to the solution of the BaumannOden method.

We proceed by considering both methods for the diffusion part of the equation and boundary conditions as in (5.2). Then the discrete formulation is also given by (5.4), except that the Dirichlet boundary condition at $\{(1, y) \mid 0<y<1\}$ is replaced by the homogeneous Neumann boundary condition $\mathbf{n} \cdot \nabla u=0$. The corresponding solutions of the symmetric and the Baumann-Oden method are shown in Fig. 17. Now the solutions are not symmetric. Again, the solution of the symmetric DG-method is poor compared to the solution of the Baumann-Oden DG method.
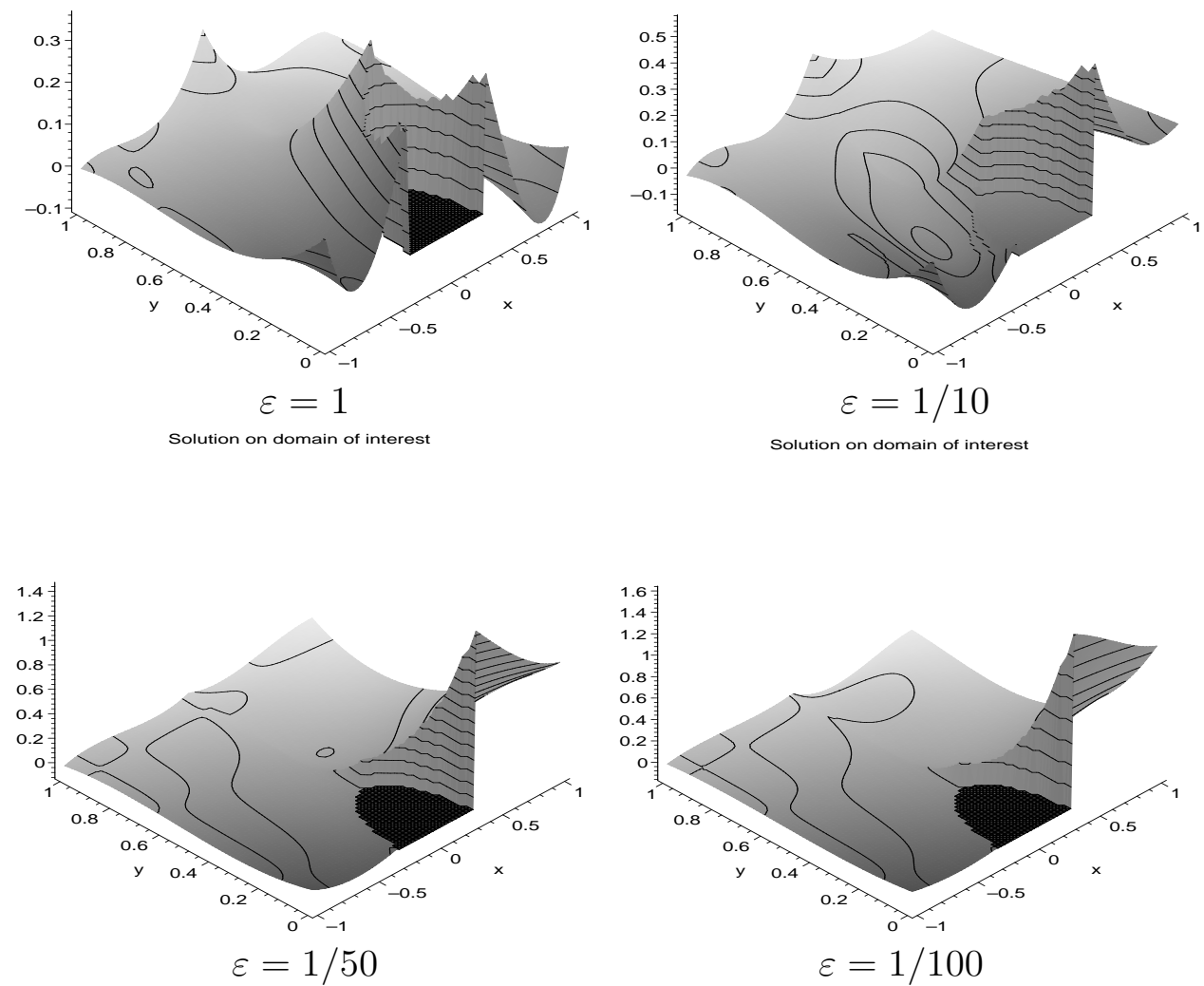

Figure 18. The approximate solution $u_{h}$ of $-\varepsilon \Delta u+u_{x}=0$, on the domain exterior of the circle for a fourth-order Baumann-Oden DG discretization with a Dirichlet boundary condition $u_{0}=1$ on the circle, $(R=3 / 10)$

Finally, we take the convection part of (5.1). The Lagrange weak formulation of the convection term for the cell $\widehat{\Omega}_{2}$ reads: find $u \in H^{1}\left(\widehat{\Omega}_{2}\right)$ and $\bar{p} \in H^{1 / 2}\left(\partial \Omega_{\text {in }}\right)$ such that

$$
\begin{array}{rlrl}
-(\nabla v \cdot \mathbf{b}, u)_{\widehat{\Omega}_{2}}+\oint_{\partial \widehat{\Omega}_{2}} v \mathbf{n} \cdot \mathbf{b} u d s+\langle\bar{p} \mathbf{n} \cdot \mathbf{b}, v\rangle_{\partial \Omega_{\mathrm{in}}} & =0 & & \forall v \in H^{1}\left(\widehat{\Omega}_{2}\right), \\
\langle q \mathbf{n} \cdot \mathbf{b}, u\rangle & =\left\langle q \mathbf{n} \cdot \mathbf{b}, u_{0}\right\rangle & \forall q \in H^{1 / 2}\left(\partial \Omega_{\text {in }}\right),
\end{array}
$$


with $\mathbf{b}=(1,0)$ and $u_{0}=1$ on the embedded circle, $\Gamma_{D} \cap \partial \Omega_{\text {in }}$, while $u_{0}=u^{-}$on the common interior boundary, $\Gamma_{\text {int }} \cap \partial \Omega_{\text {in }}$, with $u^{-}$being the upwind value of $u$ obtained from $\widehat{\Omega}_{1}$.

As for the diffusion term, we can rewrite (5.5) in a hybrid formulation, where the Lagrange multiplier is computed on $\partial \widehat{\Omega}_{\text {in }}-$ the inflow edge of the domain $\widehat{\Omega}_{2}$. Thus, we obtain: find $u \in H^{1}\left(\widehat{\Omega}_{2}\right)$ and $\widetilde{p} \in H^{1 / 2}\left(\partial \widehat{\Omega}_{\text {in }}\right)$ such that

$$
\begin{aligned}
-(\nabla v \cdot \mathbf{b}, u)_{\widehat{\Omega}_{2}}+\oint_{\partial \widehat{\Omega}_{2}} v \mathbf{n} \cdot \mathbf{b} u d s+\langle\widetilde{p} \mathbf{n} \cdot \mathbf{b}, v\rangle_{\partial \widehat{\Omega}_{\mathrm{in}}}=0 & \forall v \in H^{1}\left(\widehat{\Omega}_{2}\right), \\
\langle q \mathbf{n} \cdot \mathbf{b}, u\rangle_{\partial \Omega_{\mathrm{in}}}=\left\langle q \mathbf{n} \cdot \mathbf{b}, u_{0}\right\rangle_{\partial \Omega_{\mathrm{in}}} & \forall q \in H^{1 / 2}\left(\partial \Omega_{\mathrm{in}}\right) .
\end{aligned}
$$

Next, the second term in (5.6) is split into an integration part over the inflow and a part over the outflow edge of $\partial \widehat{\Omega}_{2}$ so that we can combine the integration of $u$ and $\widetilde{p}$ over the inflow wall $\partial \widehat{\Omega}_{\text {in }}$. This yields: find $u \in H^{1}\left(\widehat{\Omega}_{2}\right)$ and $p \in H^{1 / 2}\left(\partial \widehat{\Omega}_{\text {in }}\right)$ such that

$$
\begin{aligned}
-(\nabla v \cdot \mathbf{b}, u)_{\widehat{\Omega}_{2}} & +\langle(\widetilde{p}+u) \mathbf{n} \cdot \mathbf{b}, v\rangle_{\partial \widehat{\Omega}_{\mathrm{in}}}+\langle u \mathbf{n} \cdot \mathbf{b}, v\rangle_{\partial \widehat{\Omega}_{\mathrm{out}}}+\langle q \mathbf{n} \cdot \mathbf{b}, u\rangle_{\partial \Omega_{\mathrm{in}}} \\
& =\left\langle q \mathbf{n} \cdot \mathbf{b}, u_{0}\right\rangle_{\partial \Omega_{\mathrm{in}}} \quad \forall v \in H^{1}\left(\widehat{\Omega}_{2}\right), \forall q \in H^{1 / 2}\left(\partial \Omega_{\mathrm{in}}\right) .
\end{aligned}
$$

Writing $p=\widetilde{p}+u$, this is simplified to: find $u \in H^{1}\left(\widehat{\Omega}_{2}\right)$ and $p \in H^{1 / 2}\left(\partial \widehat{\Omega}_{\text {in }}\right)$ such that

$$
\begin{aligned}
-(\nabla v \cdot \mathbf{b}, u)_{\widehat{\Omega}_{2}} & +\langle p \mathbf{n} \cdot \mathbf{b}, v\rangle_{\partial \widehat{\Omega}_{\mathrm{in}}}+\langle u \mathbf{n} \cdot \mathbf{b}, v\rangle_{\partial \widehat{\Omega}_{\mathrm{out}}}+\langle q \mathbf{n} \cdot \mathbf{b}, u\rangle_{\partial \Omega_{\mathrm{in}}} \\
& =\left\langle q \mathbf{n} \cdot \mathbf{b}, u_{0}\right\rangle_{\partial \Omega_{\mathrm{in}}} \quad \forall v \in H^{1}\left(\widehat{\Omega}_{2}\right), \forall q \in H^{1 / 2}\left(\partial \Omega_{\mathrm{in}}\right) .
\end{aligned}
$$

To eliminate the Lagrange multiplier $p$, we recognize this function as the value of $u$ at the boundary $\partial \widehat{\Omega}_{\text {in }}$. The corresponding equations are eliminated by taking $q=\sigma v$ on $\partial \Omega_{\text {in }}$, yielding the DG-formulation of the convection term: find $u \in H^{1}\left(\widehat{\Omega}_{2}\right)$ such that

$$
\begin{gathered}
-(\nabla v \cdot \mathbf{b}, u)_{\widehat{\Omega}_{2}}+\oint_{\partial \widehat{\Omega}_{2}} v \mathbf{n} \cdot \mathbf{b} u d s+\sigma\left\langle v \mathbf{n} \cdot \mathbf{b},\left(u-u^{-}\right)\right\rangle_{\Gamma_{\mathrm{int}}}+\sigma\langle v \mathbf{n} \cdot \mathbf{b}, u\rangle_{\Gamma_{D}} \\
=\sigma\left\langle v \mathbf{n} \cdot \mathbf{b}, u_{0}\right\rangle_{\Gamma_{D}} \quad \forall v \in H^{1}\left(\widehat{\Omega}_{2}\right) .
\end{gathered}
$$

In the cell $\Omega_{1}$, the embedded boundary is an outflow boundary and, therefore, for the convection part there is no boundary condition. Therefore, we may treat $\widehat{\Omega}_{1}$ as a normal convection DG-cell.

The discretization of problem (5.1) is obtained by combining (5.4), (5.7), and (5.8): find $u_{h} \in S_{h}(\widehat{\Omega})$ such that

$$
\begin{aligned}
\left(\varepsilon \nabla u_{h}, \nabla v_{h}\right)_{\widehat{\Omega}} & -\left\langle\mathbf{n} \cdot \varepsilon \nabla u_{h}, v_{h}\right\rangle_{\partial \widehat{\Omega}}+\sigma\left\langle\mathbf{n} \cdot \varepsilon \nabla v_{h}, u_{h}\right\rangle_{\partial \Omega \cup \Gamma_{D}}-\left\langle\left\langle\varepsilon \nabla u_{h}\right\rangle,\left[v_{h}\right]\right\rangle_{\widehat{\Gamma}_{\mathrm{int}}} \\
& +\sigma\left\langle\left\langle\varepsilon \nabla v_{h},\left[u_{h}\right]\right\rangle\right\rangle_{\Gamma_{\mathrm{int}}}-\left(\nabla v_{h} \cdot \mathbf{b}, u_{h}\right)_{\widehat{\Omega}}+\left\langle v_{h} \mathbf{n} \cdot \mathbf{b}, u_{h}\right\rangle_{\partial \widehat{\Omega}_{1, \text { out }}} \\
& +\oint_{\partial \widehat{\Omega}_{2}} v_{h} \mathbf{n} \cdot \mathbf{b} u_{h} d s+\sigma\left\langle v_{h} \mathbf{n} \cdot \mathbf{b},\left(u_{h}-u_{h}^{-}\right)\right\rangle_{\Gamma_{\mathrm{int}}}+\sigma\left\langle v_{h} \mathbf{n} \cdot \mathbf{b}, u_{h}\right\rangle_{\Gamma_{D} \cap \partial \Omega_{2}} \\
& =\sigma\left\langle\mathbf{n} \cdot \varepsilon \nabla v_{h}, 1\right\rangle_{\Gamma_{D}}+\sigma\left\langle v_{h} \mathbf{n} \cdot \mathbf{b}, 1\right\rangle_{\Gamma_{D} \cap \partial \Omega_{2}} \quad \forall v_{h} \in S_{h}(\widehat{\Omega}) .
\end{aligned}
$$

Fig. 18 shows the solutions of the fourth-order discretization of (5.9) for $R=3 / 10$, for different values of $\varepsilon=1,0.1,0.02,0.01$. We see that in all cases the solution is stable. For values of $\varepsilon=O(1)$ we clearly see the approximation of the boundary condition $u_{0}=1$ on the 
circle bow, while for small values of $\varepsilon$, when the true solution shows a thin boundary layer, typical effects of the weak boundary requirement show up. Fig. 19 shows the solution for $\varepsilon=1 / 50$. Although it seems that the solution is not able to catch the boundary layer in $\Omega$ on the upwind side of the circle, we clearly see a boundary layer arising in the fictitious part of the domain if we consider the total $\widehat{\Omega}$. Clearly, the cubics are not able to represent the thin circular boundary layer. Note, in particular, that at $x=0, y<R$, there is a discontinuity in the fictitious part $\widetilde{\Omega}$. For small values of $\varepsilon<<1$ the boundary layer disappears.

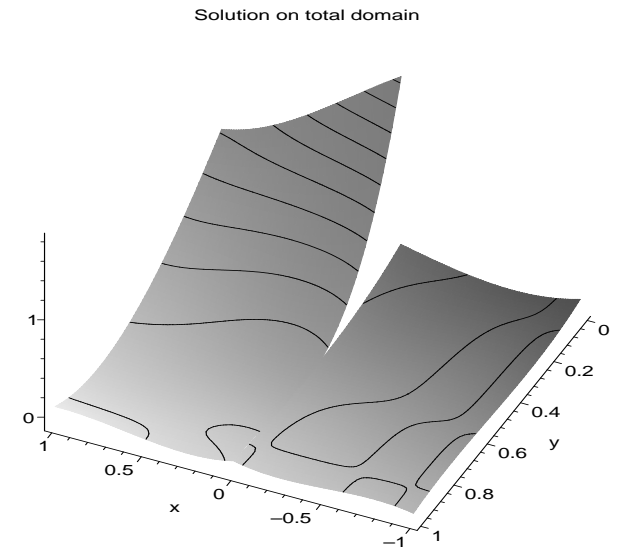

Total domain

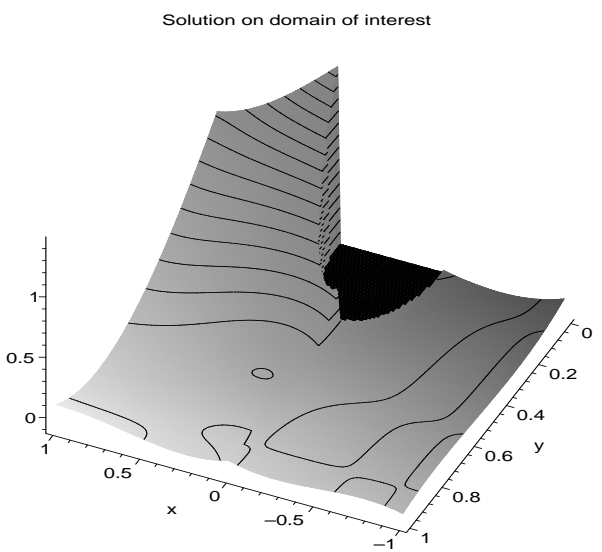

Domain of interest

Figure 19. The approximate solution $u_{h}$ for the same problem as in Fig. 18, with $\varepsilon=1 / 50$. At the right, the approximate solution on $\Omega$, the domain of interest. Left, the approximate solution on $\widehat{\Omega}$, the whole domain where the approximation is constructed, including the fictitious part

\section{Conclusions}

In this paper, we propose a technique for treating second-order elliptic PDEs with complex Dirichlet boundary conditions in combination with a discontinuous Galerkin discretization. The aim is to maintain the structured, regular rectangular grid while solving problems with irregular curved boundary conditions.

The complex domain on which the solution is sought is covered by a fictitious domain with a structured, regular rectangular grid. An embedded boundary is the transition between the domain of interest and the fictitious part of the computational domain.

We present and compare several weak forms for the diffusion part of the equation: the Lagrange multiplier form, the hybrid form, and the DG-form. The hybrid form shows stability for an arbitrary location of the embedded boundary, whereas for the other forms the discretization may become unstable for particular locations of the Dirichlet BC. The problem is studied first for a single cell and then for a couple of adjacent cells, either sharing or not sharing the embedded boundary. We also describe the treatment of the convection part in the equation.

As an example, we solve a singularly perturbed boundary value problem on a complex domain by means of a fourth-order DG-discretization on only two cells. Although, as expected, it appears to be impossible to accurately represent sharp boundary layers with a complex structure by means of a few cubic polynomials, the boundary condition treatment is quite effective in handling such complex situations. 


\section{References}

[1] D. N. Arnold, F. Brezzi, B. Cockburn, and D. Marini, Unified analysis of discontinuous Galerkin methods for elliptic problems, SIAM J. Numer. Anal., (2002), pp. 1749-1779.

[2] R. Cortez and M. Minion, The BLOB projection method for immersed boundary problems, J. Comput. Phys., 161 (2000), pp. 428-453.

[3] D. Goldstein, R. Handler, and L. Sirovich, Modeling a no-slip flow boundary with an external flow field, J. Comp. Phys., 105 (1993), pp. 354-366.

[4] W. Hackbusch, Theorie und Numerik Elliptischer Differentialgleichungen, Teubner Studienbuecher, 1986.

[5] P. W. Hemker, A singularly perturbed model problem for numerical computation, J. Comp. Appl. Math., 76 (1997), pp. 277-285.

[6] P. W. Hemker, W. Hoffmann, and M. H. van Raalte, Two-level Fourier Analysis of a multigrid Approach for Discontinuous Galerkin Discretisation, Tech. Rep. MAS-R0206, CWI, Amsterdam, 2002, To appear in SIAM SISC.

[7] D. W. Hewett, The embedded curved boundary method for orthogonal simulation meshes, J. Comp. Phys., 138 (1997), pp. 585-616.

[8] D. W. Hewett and C. S. Kueny, The Dielectric Boundary Condition for the Embedded Curved Boundary (ECB) Method, Tech. Rep. UCRL-JC-129703, Lawrence Livermore Nat. Lab., 1998, presented at the 16th International Conference on the Numerical Simulation of Plasmas, February 1998, Santa Barbara, CA.

[9] J. Kim, D. Kim, and H. Choi, An immersed-boundary finite-volume method for simulations of flow in complex geometries, J. Comput. Phys., 171 (2001), pp. 132-150.

[10] C. S. Kueny, Embedded Curved Boundaries and Adaptive Mesh refinement, Tech. Rep. UCRL-JC129729, Lawrence Livermore Nat. Lab., 1998.

[11] R. J. LeVeque and Z. Li, The immersed interface method for elliptic equations with discontinuous coefficients and singular sources, SIAM J. Numer. Anal., 31 (1994), No. 4, pp. 1019-1044.

[12] R. J. LeVeque and Z. Li, Immersed interface methods for Stokes flow with elastic boundaries or surface tension, SIAM J. Sci. Comput., 18 (1997), No. 3, pp. 709-735.

[13] Z. Li, The Immersed Interface Method - A Numerical Approach for Partial Differential Equations with Interfaces, Ph.D. thesis, University of Washington, 1994.

[14] J. Nitsche, Über ein Variationsprinzip zur Lösung von Dirichlet Problemen bei Verwendung von Teilräumen die keinen Randbedingungen unterworfen sind, Abh. Math. Semin. Univ. Hamb., 36 (1971), p. 9.

[15] C. S. Peskin, Numerical analysis of blood flow in the heart, J. Comp. Phys., 25 (1977), pp. $220-252$.

[16] C. Tu and C. S. Peskin, Stability and instability in the computation of flows with moving immersed boundaries: A comparison of three methods, SIAM J. Sci. Comput., 13 (1992), pp. 1361-1376. 